\title{
Human CRY1 variants associate with attention deficit/hyperactivity disorder
}

\author{
O. Emre Onat, ${ }^{1}$ M. Ece Kars, ${ }^{1}$ Şeref Cül, ${ }^{2,3}$ Kaya Bilguvar, ${ }^{4}$ Yiming Wu, ${ }^{5}$ Ayşe Özhan, ${ }^{1}$ Cihan Aydın, ${ }^{2,3}$ A. Nazlı Başak, ${ }^{6}$ \\ M. Allegra Trusso, ${ }^{7}$ Arianna Goracci, ${ }^{7}$ Chiara Fallerini, ${ }^{8}$ Alessandra Renieri, ${ }^{8,9}$ Jean-Laurent Casanova, ${ }^{10,11,12,13,14}$ \\ Yuval Itan, ${ }^{5,15}$ Cem E. Atbaşoğlu, ${ }^{16}$ Meram C. Saka, ${ }^{16}$ i. Halil Kavaklı, ${ }^{2}$ and Tayfun Özçelik ${ }^{1,7,18}$
}

\begin{abstract}
'Department of Molecular Biology and Genetics, Bilkent University, Ankara, Turkey. ${ }^{2}$ Department of Chemical and Biological Engineering and ${ }^{3}$ Department of Molecular Biology and Genetics, Koç University, Istanbul, Turkey. ${ }^{4}$ Department of Cenetics, Yale Center for Cenome Analysis, Yale University School of Medicine, New Haven, Connecticut, USA. ${ }^{5}$ Charles Bronfman Institute for Personalized Medicine, Icahn School of Medicine at Mount Sinai, New York, New York, USA. ${ }^{6}$ Neurodegeneration Research Laboratory, Suna and Inan Kıraç Foundation, KUTTAM, Koç University, Istanbul, Turkey. ${ }^{7}$ Division of Psychiatry, Department of Molecular Medicine and Development, Azienda Ospedaliera Universitaria Senese, Siena, Italy. ${ }^{8}$ Medical Genetics, University of Siena, Siena, Italy. ${ }^{9}$ Cenetica Medica, Azienda Ospedaliera Universitaria Senese, Siena, Italy. ${ }^{10}$ St. Giles Laboratory of Human Genetics of Infectious Diseases, Rockefeller Branch, Rockefeller University, New York, New York, USA. "Laboratory of Human Genetics of Infectious Diseases, Necker Branch INSERM U1163, Necker Hospital for Sick Children, Paris, France. ${ }^{2}$ Imagine Institute, University of Paris, Paris, France. ${ }^{13}$ Pediatric Immunology-Hematology Unit, Necker Hospital for Sick Children, Paris, France. ${ }^{14}$ Howard Hughes Medical Institute (HHMI), Rockefeller University, New York, New York, USA. ${ }^{15}$ Department of Cenetics and Genomic Sciences, Icahn School of Medicine at Mount Sinai, New York, New York, USA. ${ }^{15}$ Department of Psychiatry, Ankara University Medical School, Ankara, Turkey. ${ }^{17}$ Neuroscience Program, Graduate School of Engineering and Science, and
\end{abstract} ${ }^{18}$ Institute of Materials Science and Nanotechnology, National Nanotechnology Research Center (UNAM), Bilkent University, Ankara, Turkey.

\begin{abstract}
Attention deficit/hyperactivity disorder (ADHD) is a common and heritable phenotype frequently accompanied by insomnia, anxiety, and depression. Here, using a reverse phenotyping approach, we report heterozygous coding variations in the core circadian clock gene cryptochrome 1 in 15 unrelated multigenerational families with combined ADHD and insomnia. The variants led to functional alterations in the circadian molecular rhythms, providing a mechanistic link to the behavioral symptoms. One variant, CRV1 11 c.1657+3A >C, is present in approximately $1 \%$ of Europeans, therefore standing out as a diagnostic and therapeutic marker. We showed by exome sequencing in an independent cohort of patients with combined $A D H D$ and insomnia that 8 of 62 patients and 0 of 369 controls carried CRY1 111 . Also, we identified a variant, CRY1 6 c. $825+1 \mathrm{C}>\mathrm{A}$, that shows reduced affinity for BMAL1/CLOCK and causes an arrhythmic phenotype. Genotype-phenotype correlation analysis revealed that this variant segregated with ADHD and delayed sleep phase disorder (DSPD) in the affected family. Finally, we found in a phenome-wide association study involving 9438 unrelated adult Europeans that CRY1D11 was associated with major depressive disorder, insomnia, and anxiety. These results defined a distinctive group of circadian psychiatric phenotypes that we propose to designate as "circiatric" disorders.
\end{abstract}

\section{Introduction}

Sleep is genetically regulated by the circadian rhythm. Disruption of this rhythm leads to aberrant sleep patterns (1-4). Sleep is also frequently disturbed in individuals with psychiatric disorders. For example, dim light melatonin onset, a reliable marker of circadian function, is delayed in children with attention deficit/hyperactivity disorder (ADHD) $(5,6)$. However, a causal relationship between inherited circadian mutations and psychiatric traits has not been established (7-12). More generally, psychiatric and sleep disorders proved to be extremely difficult to solve by classic phenotype-first studies because of complex factors that are at both the phenotype and genotype definition and characterization levels. For example, phenotype misclassifications arise from problems of distinguishing health from disease, the episodic nature

Conflict of interest: The authors have declared that no conflict of interest exists. Copyright: () 2020, American Society for Clinical Investigation.

Submitted: December 5, 2019; Accepted: April 16, 2020; Published: June 15, 2020

Reference information: J Clin Invest. 2020;130(7):3885-3900.

https://doi.org/10.1172/JCl135500. of symptoms, and establishing accurate diagnostic criteria. Likewise, interpretation of genotype data becomes a challenge because of early postzygotic mutations, incomplete penetrance, variable expressivity, or high levels of genetic heterogeneity (11, 13). Several levels of causation have been implicated in the emergence of heterogeneity: (a) the existence of many different rare and severe mutations of the same gene in unrelated individuals, (b) the same mutation leading to different phenotypic outcomes in different individuals, (c) mutations in different genes leading to the same disorder, and (d) a collective effect of many individual gene events (14). As a result, the genomic landscape of ADHD and more generally of psychiatric disorders remains largely unknown, and much of the genetic risk is unexplained.

Reverse phenotyping is an alternative approach to overcome the uncertainties inherent to clinical diagnoses in the patient care setting and promises to achieve accurate phenotype assignments in the research setting (15-18). Also termed the genotype-first approach, reverse phenotyping consists of 3 consecutive steps: (a) collection of genomic data and candidate discovery, (b) determination of causality by phenotype and segregation analyses in 


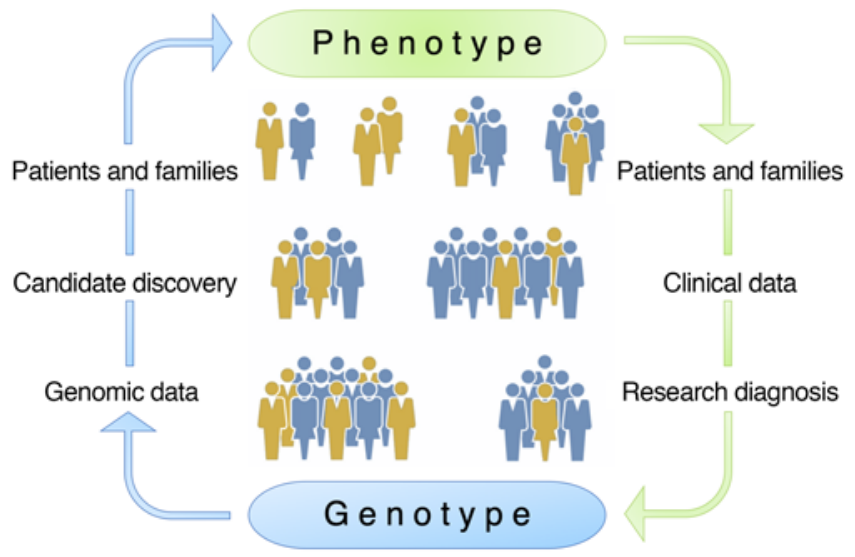

families, and (c) population screening for the mutant locus in phenotypically well-characterized cohorts (Figure 1).

In this context, using reverse phenotyping, we recently identified a gain-of-function CRY1 variant (CRY1 $11, C R Y 1$ c. $1657+3 A>C$, rs184039278) that provides a mechanistic link to delayed sleep phase disorder (DSPD), a common form of insomnia, in 6 large multigenerational Turkish families (1). CRY1 is an essential component of the core molecular clock and represses the activity of the transcription factors CLOCK and BMAL1 transactivation (19). As we observed a high incidence of behavioral endophenotypes including a history of depression mainly in mutation carriers, we decided to further characterize the clinical features of individuals from these 6 families, as well as individuals from 6 additional Turkish families with DSPD.

\section{Results}

Reverse phenotyping in the discovery cohort. We first performed clinical evaluations of a cohort of 96 individuals from 12 families from Turkey. A systematic psychiatric assessment was conducted using the American Psychiatric Association's Diagnostic and Statistical Manual of Mental Disorders (DSM-5, 5th edition) (20) and questionnaires $(21,22)$. For this evaluation, 2 board-certified psychiatrists with extensive experience in psychiatric analysis, interviewed the study participants without knowing their genotype status. The interviews with each study participant were conducted over a period of 60-90 minutes and were designed to ascertain the psychosocial, functional, and mental status of each of the subjects. The ADHD Child Evaluation (ACE) interview questionnaires "ACE - A diagnostic interview of ADHD in children" and "ACE+ - A diagnostic interview of ADHD in adults" were used to support the interviews (http://www.psychology-services.uk.com). A polysomnographic sleep recording of a CRY1 11 carrier using continuous electroencephalography, electromyography, and electrooculography was also done and is reported elsewhere (1).

This comprehensive phenotyping revealed that the symptoms and signs that define ADHD were present in addition to DSPD in 46 of 48 mutation-positive individuals and absent in 44 of 48 mutation-negative relatives or spouses. Two carriers and 3 WT individuals were classified as ADHD spectrum and $1 \mathrm{WT}$ individual as affected. This corresponded to a total of 47 affected individuals, 37 of whom displayed patterns of behavior consistent with a com-
Figure 1. Reverse phenotyping. Schematic of the phenotype-first (green) versus the genotype-first (also referred to as reverse phenotyping, blue) approaches for identification of causal gene mutations. The phenotype-first approach relies on identification of patients and families, collection of clinical data, accurate research diagnosis, and, finally, collection of genotype data steps. In the genotype-first approach, the process is reversed and starts with the analysis of genomic data and selection of candidate variants followed by comprehensive clinical phenotyping of patients and families to make accurate genotype-phenotype correlations.

bined presentation, 2 who were found to be predominantly hyperactive and impulsive, and 8 who were found to be predominantly inattentive. We independently confirmed our observations by characterizing 5 mutation carriers from 2 Italian families using the criteria described above for the Turkish families (Figure 2). This evaluation was carried out by 2 board-certified psychiatrists. All mutation carriers were diagnosed with $\mathrm{ADHD}$, and 3 carriers also experienced sleep disturbances (Figure 3, A-N, and Supplemental Tables 1-3; supplemental material available online with this article; https://doi.org/10.1172/JCI135500DS1). Visual inspection of segregation patterns in the pedigrees suggested an autosomal dominant inheritance of the phenotype, and no significant difference in ADHD and DSPD symptoms was observed between homozygous and heterozygous carriers. These results strongly suggested that circadian dysfunction, as exemplified by the CRY1 11 mutation, has a very strong association with combined ADHD and DSPD (OR $281, P=1.99 \times 10^{-21}$, Fisher's exact test).

ADHD comorbidities. ADHD is possibly an extreme expression of continuous heritable traits significantly correlated with educational outcomes, psychiatric or personality disorders, obesity-related phenotypes, smoking or smoking-related cancer, reproductive success, longevity, and insomnia (12). Therefore, we searched for ADHD comorbidities in the families using the phenotype information documented during the examination, which included demographics, history of depression, and smoking status. We documented an overrepresentation of a recurrent history of depression in CRY1 $\triangle 11^{+}$ adults (34 of $53,64.2 \%$; $n=4$ homozygous, $n=30$ heterozygous) compared with $C R Y 1 \Delta 11^{-}$adults (5 of $48,10.4 \%$; OR $15.4, P=1.65$ $\times 10^{-8}$ ) (Supplemental Table 4). These findings are consistent with epidemiological studies, which report that ADHD may sometimes be the underlying cause for features of clinical depression, especially in adults (23). Also, we documented an increase in smoking (ever vs. never) in CRY1 $\triangle 11$ carriers compared with their WT family members ( $83 \%$ vs. $43 \%$ in males and $46 \%$ vs. $33 \%$ in females). According to the 2016 Global Adult Tobacco Survey in Turkey (24), this increase holds when compared with the general Turkish population (44\% in males and $19 \%$ in females), and warrants further investigation in a larger cohort (Supplemental Table 4) and warrants further investigation in a larger cohort (Supplemental Table 4).

Sunlight exposure. Epidemiological studies suggest a link between sleep, mood, and sunlight. Clinicians used bright light 


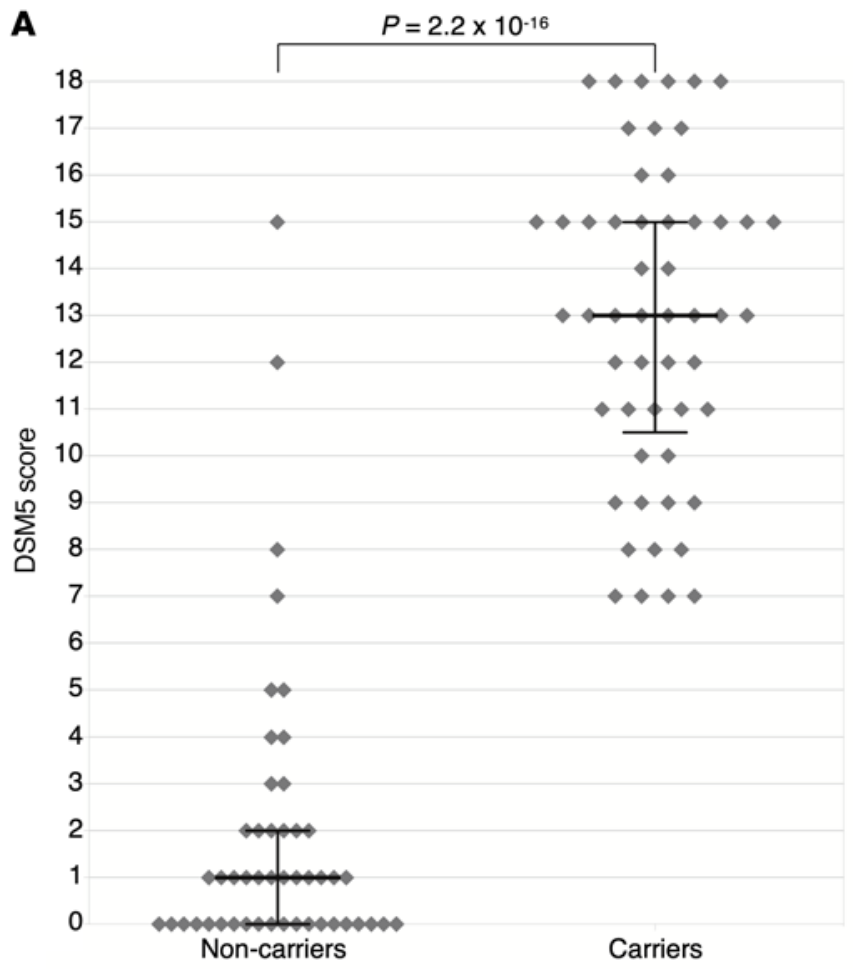

B

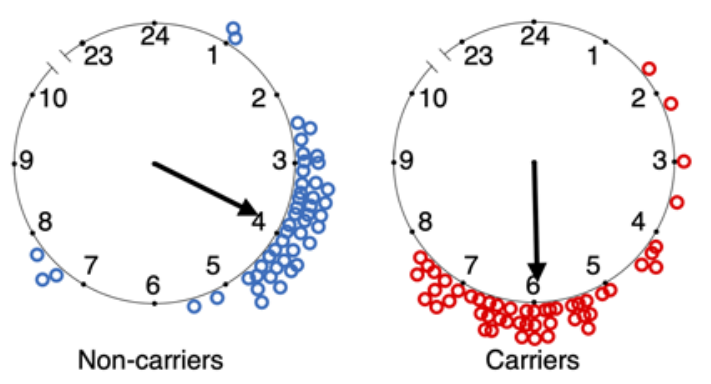

$(25,26)$ or high solar intensity $(27)$ as a controlled intervention for circadian rhythm sleep problems as well as ADHD. We performed a systematic analysis of direct sunlight exposure durations in the 52 CRY1 11 carriers of these 14 families, and investigated the correlation with mid-sleep points and ADHD severity as judged by clinical observations and questionnaire scores (Supplemental Table 5). Although we did not observe a correlation for mid-sleep points, we noted a milder presentation of ADHD in 33 individuals, 19 of whom had longer periods of daily sunlight exposure compared with the 19 individuals with severe ADHD. A scatter plot of Adult ADHD Self-Report Scale (ASRS) scores and the mean duration of sun exposure revealed a moderate negative correlation (Figure 4, Spearman's rho $=-0.46, P=0.0005$ ). This observation highlights the potential value of light in addition to medications and talk therapies in the management of ADHD (25-27). Accordingly, we propose including the Munich ChronoType Questionnaire (MCTQ) (21) as part of any ADHD diagnostic evaluation to document sunlight exposure durations and sleep.

CRY1 11 penetrance and ADHD phenotype heterogeneity. High degrees of allelic or locus heterogeneity, a presence of phenocopies, and, most important, difficulties inherent to psychiatric
Figure 2. DSM-5 ADHD scores, mid-sleep point on free days, and CRY1D11 mutation status of the 14-family discovery cohort. (A) Each dot represents the DSM- 5 score of 101 individuals. Medians and interquartile ranges are marked for $53 C R Y 1 \Delta 11$ carriers and 48 intrafamilial noncarriers. A Mann-Whitney $U$ test indicated that the DSM- 5 score for the carriers (median $=13$ ) was greater than that for the noncarriers (median $=1$ ). $U$ $=64.5, P=2.2 \times 10^{-16}$. (B) The mid-sleep point on free days (MSF) for the same subjects are plotted on a discontinuous clock face from 2300 to 1000 hours for noncarriers (left, blue) and carriers (right, red). No subject data fell within the gap time (1000 to 2300 hours) not represented in the plot.

nosological definitions - especially due to the fluctuating nature of particular manifestations in an individual evaluated at specific time points - pose major challenges in identifying the genomic determinants of complex disorders $(16-18,28,29)$, and ADHD is no exception. Two individuals from the Turkish families, who were heterozygous for CRY1 11 , displayed an ADHD spectrum of symptoms but failed to fulfill the DSM- 5 criteria. We also noted that 3 Turkish individuals from the families had signs and symptoms of ADHD, yet were WT for CRY1 111 (Figures 2 and 3, and Supplemental Table 1). For example, in the Turkish cohort, patient 17-010, who is a highly successful student preparing for medical school exams, had a relatively high score on the ASRS self-reporting questionnaire, but was unlikely to have ADHD based on her clinical evaluation. Her condition was more consistent with an anxiety disorder or performance anxiety. We made a similar observation for subject 17-091, who had a borderline DSM-5 score but, based on the clinical evaluation, was unlikely to have ADHD. This individual's symptoms were secondary to a recent coronary artery bypass surgery. Both of these individuals had normal sleep patterns. Therefore, these 2 subjects and an additional subject, 16-082, were classified as probably not affected. However, 17-368 and 16-027, who are carriers, were classified as probably affected on the basis of clinical observations indicating that they could be in partial remission.

Phenotype-first approach. To complement the reverse phenotyping, we selected 447 unrelated adults from our in-house database of Turkish families with obesity/metabolic phenotypes and designated them as the validation cohort (Supplemental Figure 1, $\mathrm{A}-\mathrm{C})$. We next reviewed their medical charts and contacted all of them for MCTQ and ASRS questionnaire evaluations $(21,22)$. Supplemental Table 6 presents the demographic data for this cohort (i.e., age, sex, education, occupation, marital status, and number of children). This database is not publicly accessible, but ethics and consent procedures for the subjects allowed for recontact. During this first step, 108 of 447 (24.2\%) individuals self-identified as having excessive inattention and/or hyperactivity and impulsivity, and 185 of 447 (41.3\%) as having delayed sleep patterns on free days. These high numbers were expected, since a majority of the families were recruited to study the genetic basis for obesity. BMI data revealed a marked positive correlation with ADHD, and obesity is associated with sleep problems (12). The next step of a physician-led interview using the DSM- 5 criteria with the 108 ASRS-positive individuals confirmed that 78 of them met the ADHD diagnostic criteria. Exclusion criteria were autism, epilepsy, intellectual disability, psychosis/schizophrenia, ADHD symptoms due to personality disorders, adoption, sexual or phys- 
A DSPD-1

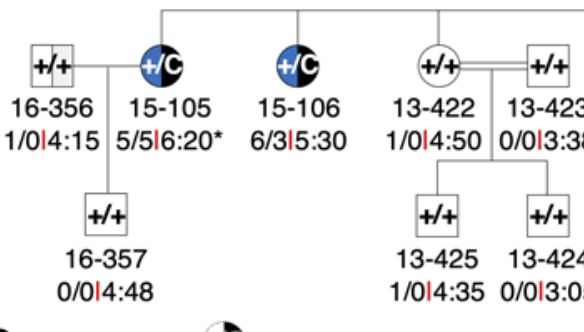

C DSPD-4
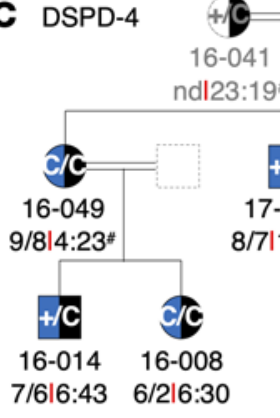

ndl23:19"

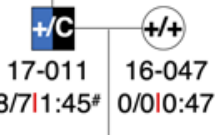

\section{$-047$}

$8 / 711: 45^{*}$ 0/010:47
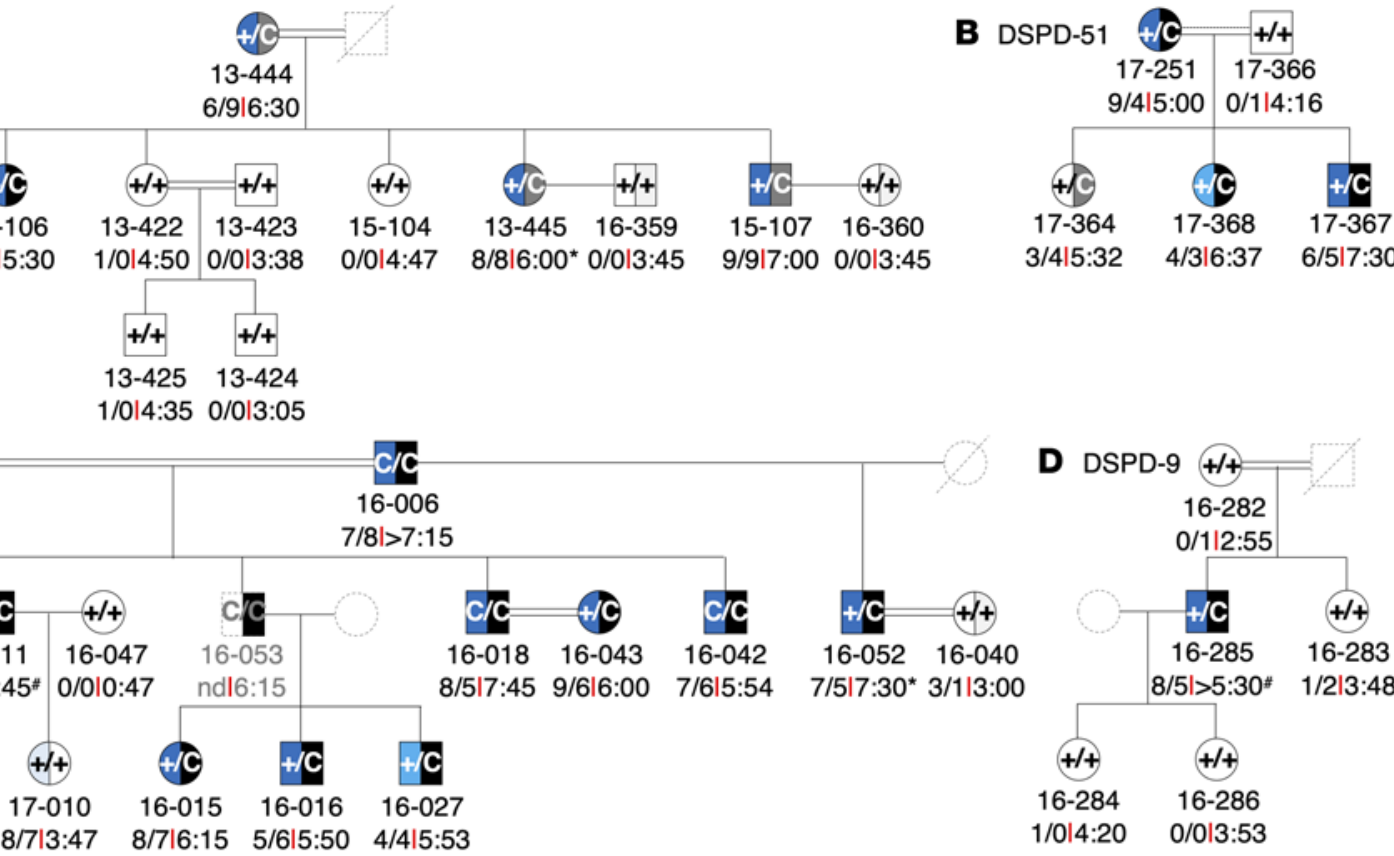

$8 / 7|3: 47 \quad 8 / 7| 6: 15 \quad 5 / 6|5: 50 \quad 4 / 4| 5: 53$
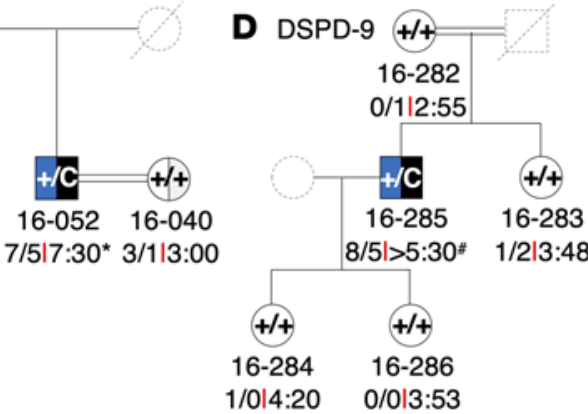

E DSPD-6

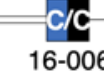

$7 / 8 \mid>7: 15$

16-073

ndl2:15

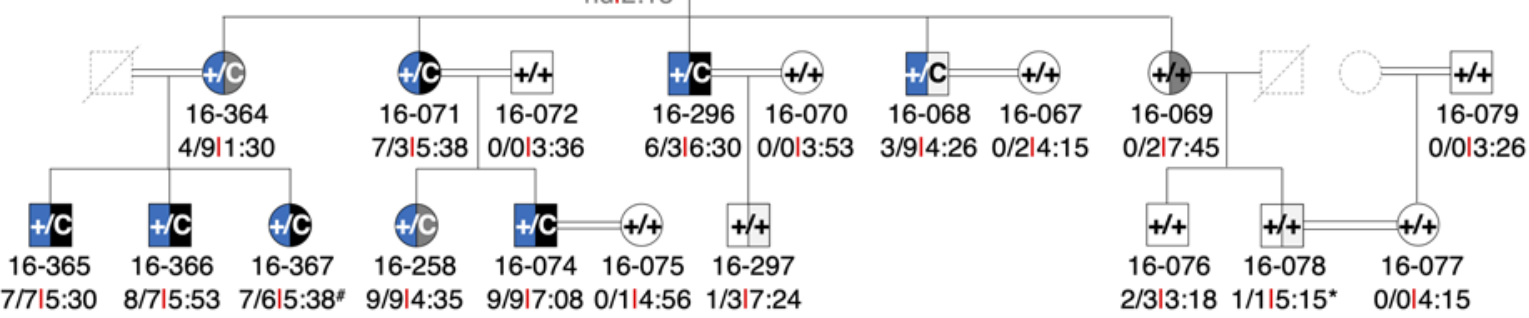

F DSPD-14

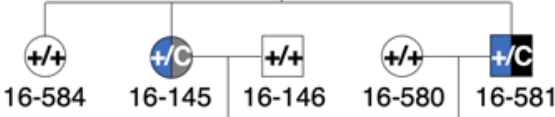

\begin{tabular}{cc|cc|c}
$16-584$ & $16-145$ & $16-146$ & $16-580$ & $16-581$ \\
\hline $0 / 0 \mid 4: 38$ & $9 / 9 \mid 3: 56^{\star}$ & $0 / 0 \mid 3: 41$ & $1 / 0 \mid 3: 11$ & $5 / 26: 49$
\end{tabular}

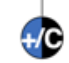

16-144

$9 / 8 \mid>5: 40$

$6 / 3 \mid>6: 05$
G DSPD-2

14-117 14-118

$5 / 7 \mid 6: 00 * 2 / 113: 00$

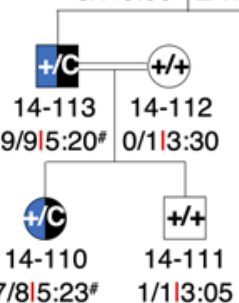

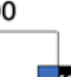

$\underset{+4-114}{+\mathbf{C}} \quad \stackrel{+/+}{14-115}$

9/916:25" 0/113:30

$+/ \mathrm{C}$

14-116

nd

\section{DSPD-7}

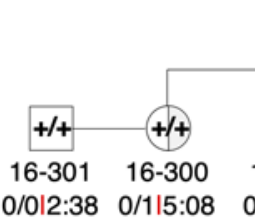

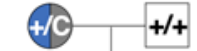

16-224 16-225

7/8|7:15 0/0|4:15

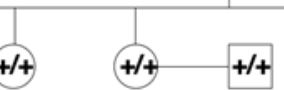

16-268 $\quad 16-302 \quad 16-303$ 0/113:32 0/0|3:47 $0 / 0 \mid 3: 40$

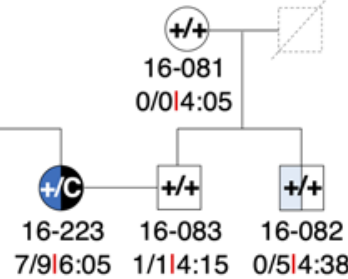

H DSPD-34
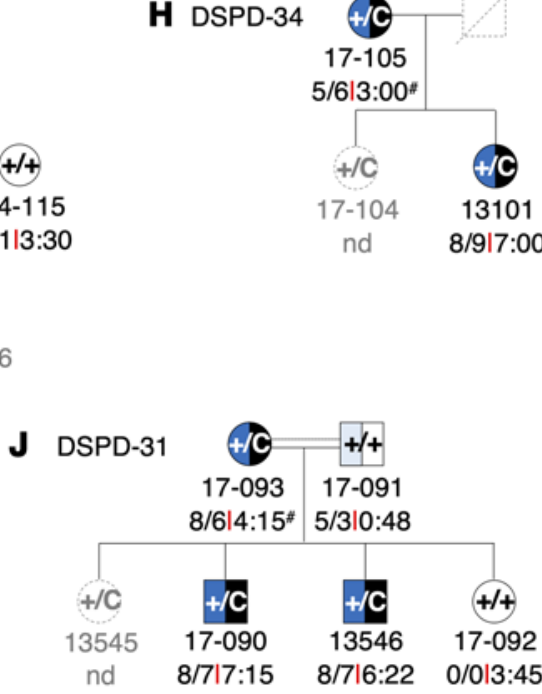

K DSPD-52

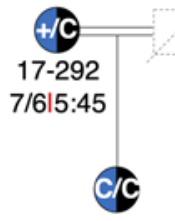

17-281

$6 / 516: 30$
L DSPD-53

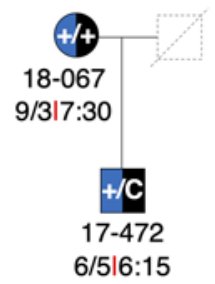

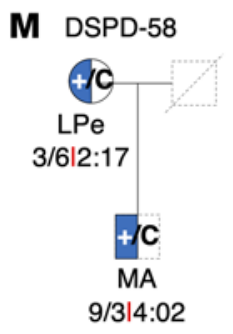

N DSPD-59

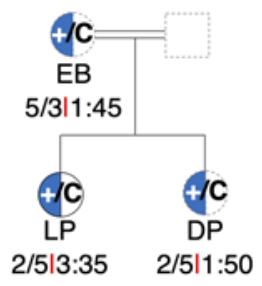

ADHD affected

ADHD probably affected (Suppl. Tables 2 and 3 )

$\square$ ADHD probably not affected (Suppl. Tables 2 and 3 )

- DSPD affected late

DSPD probably affected (Suppl. Table 4)

$\square$ DSPD probably not affected (Suppl. Table 4)

\# DSPD affected fragmented

$\square$ not affected

uninterpretable/unknown (Suppl. Tables 2-4)

* current or former shift worker 
Figure 3. DSPD and ADHD phenotypes in CRY1411 carrier families of Turkish and Italian descent. (A-N) Families DSPD-1, -2, -4, -6, -7, -9, -14, $-31,-34,-51,-52,-53,-58$, and -59 underwent psychiatric evaluation during personal interviews and using sleep and ChronoType questionnaires (1, 21, 22). Individual ID numbers followed by DSM-5 scores and the MSF are shown below and the genotype status (+ denotes the WT allele; $\mathrm{C}$ denotes the mutant allele) in the pedigree symbols. See Supplemental Tables 1-3 for further details.

ical abuse, birth weight under $1.5 \mathrm{~kg}$, and other neurological or systemic disorders that might explain ADHD symptoms $(11,13)$. Sixty-two of 78 (79.5\%) of these individuals also had DSPD (Supplemental Table 6).

Whole-exome sequencing in the validation cohort. Exome sequencing was performed for all of the 447 individuals and revealed that only CRY1 reached genome-wide statistical significance after variant prioritization using a variant-based, genebased burden analysis and an optimal unified sequence kernel association test (SKAT-O) (ref. 30, Figure 5, A-C, and Supplemental Table 7). We identified $8 C R Y 1 \Delta 11$ carriers who were classified in the combined ADHD and DSPD category and 1 carrier who had isolated DSPD. These results validate the observations in the discovery cohort, suggesting that as many as 1 in 8 ( 8 of 62, 13\%) patients with combined ADHD and DSPD and 1 in 21 (9 of $185,5 \%)$ with DSPD may carry CRY1 11 .

CRY1 c.1657+3A $>C$ allele frequency in the Turkish and Italian populations. We combined the data from 6 different databases, which corresponds to a total of 5465 individuals, and observed a Turkish minor allele frequency (MAF) of 0.0124 for CRY1 c.1657+3A $>C$ (Scientific and Technological Research Council of Turkey [TÜBİTAK]: $n=1082$; Yale Center for Genome Analysis [YCGA]: $n=1193$; Rockefeller University, Casanova Lab, Turkish individuals: $n=253$; Koç University, Başak lab, Turkish individuals: $n=1191$; Bilkent, Bilkent University database: $n=1013$; Ankara University Brain Research Center [AUBAUM]: $n=733$ ). In the Italian population, the allele frequency is 0.01678 (Network for Italian Genomes database [NIG]: $n=447$ ) (Figure 6A and Supplemental Table 8).

Age estimation of CRY1 11 in the Turkish and European populations. In the Genome Aggregation Database (gnomAD), the CRY1 11 allele frequency is $0.0044(0.03 \%-3.3 \%)$, which is, for example, 1 in 103 individuals in the European-derived general population (31). In the Turkish population, the frequency is higher (0.0124; Figure 6A and Supplemental Table 8). To determine whether all occurrences of CRY1 111 descended from a single ancestral mutational event or arose independently, we combined the haplotypes of 297 Europeans from the 1000 Genomes Project data (32) with those of the 447-individual validation cohort. We observed a shared haplotype block of $571.6 \mathrm{~kb}$, suggesting a common founder effect (Figure 7). Assuming a 25-year intergeneration interval, the age of $C R Y 1 \Delta 11$ is estimated to be approximately 11,175 years (95\% CI: 6550-13,700), corresponding to 447 generations for the Turkish population, and 6425 years ( $95 \%$ CI: 5500-9500) and 257 generations for the European population (Figure 6, B and C). Based on these data, CRY1 11 probably spread to Europe during the arrival of Neolithic Anatolian farmers approximately 8500 years ago (33).
Phenome-wide association study of CRY1 11 in the BioMe BioBank. To test whether distinct circadian psychiatric outcomes define CRY1 11 mutations, we consulted the BioMe BioBank of the Institute for Personalized Medicine at the Icahn School of Medicine at Mount Sinai for a phenome-wide association study (PheWAS). We investigated the electronic medical record-linked phenotypes (ICD-10-CM codes; Centers for Disease Control and Prevention's International Classification of Diseases, 10th Revision, Clinical Modification) across 9438 unrelated adult European-only samples from the BioMe BioBank and observed 324 $C R Y 1 \Delta 11$ carriers and 9114 noncarriers $(\mathrm{MAF}=0.017)$. The initial PheWAS did not reveal a positive association of CRY1 11 with a distinct phenotype. However, when we filtered phenotype codes that interfere with an accurate ADHD diagnosis $(11,13), 37$ of 80 individuals with ADHD were excluded, and we detected a 2.2 -fold increase in the OR (95\% CI: 0.42-6.87) for ADHD in CRY1 11 carriers with respect to the OR for controls, though the remaining sample size $(n=43)$ was not sufficient to reach statistical significance. Filtered ICD-10-CM codes correspond to major mental and neurological disorders, congenital malformations of the nervous system, chromosomal abnormalities, and endocrine and metabolic diseases that lead to intellectual impairment (Supplemental Table 9). We did not filter out ADHD comorbidities (20,34). After filtering, 238 CRY1 11 carriers and 6825 noncarriers with 6820 ICD-10-CM codes remained, and a repeat PheWAS revealed the strongest associations with major depressive disorder (MDD) (single episode: OR 1.91, $P=7.87 \times 10^{-4}$; recurrent: OR 2.55, $P=$ $\left.1.28 \times 10^{-2}\right)$; insomnia (OR 1.84, $\left.P=3.87 \times 10^{-3}\right)$; anxiety (OR 1.68, $\left.P=4.56 \times 10^{-3}\right)$; glaucoma (OR 3.65, $\left.P=7.11 \times 10^{-3}\right)$; and nicotine dependence (OR 2.01, $P=2.52 \times 10^{-2}$ ) (Table 1 and Supplemental Tables 10 and 11). Glaucoma has been reported with increased frequency in individuals with sleep problems, and an association with CRY1 11 requires further investigation (35).

Identification of $C R Y 1 \triangle 6$. Exome sequencing in the validation cohort identified 1 more individual heterozygote for a rare $C R Y 1$ variant (c.825+1G>A, rs780614131, Supplemental Table 12) in the

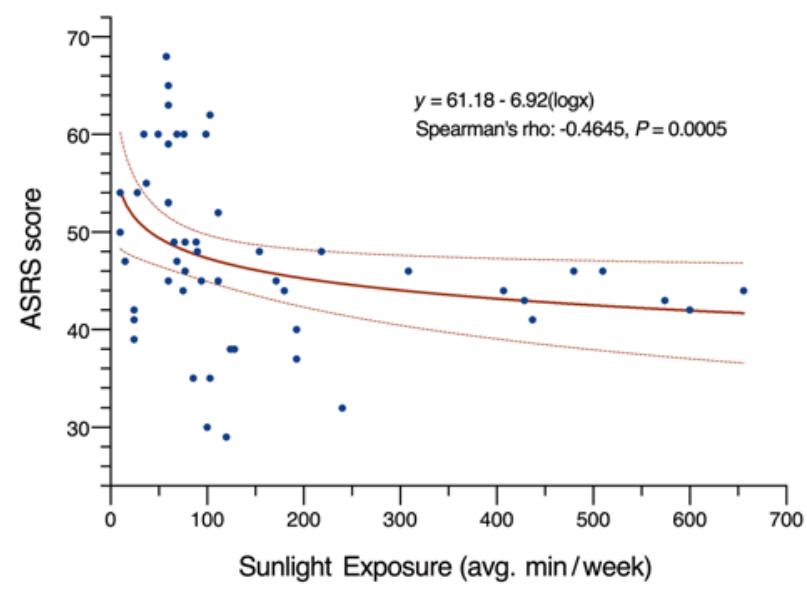

Figure 4. Plot showing the correlation between sunlight exposure and ADHD severity. The ADHD severity of 53 CRY1 11 carriers was assessed using ASRS scores, which are plotted against the mean duration of sunlight exposure in minutes per week $(R=-0.44)$. The line of best fit demonstrates the negative correlation, and the dashed lines represent the $95 \% \mathrm{Cls}$. 

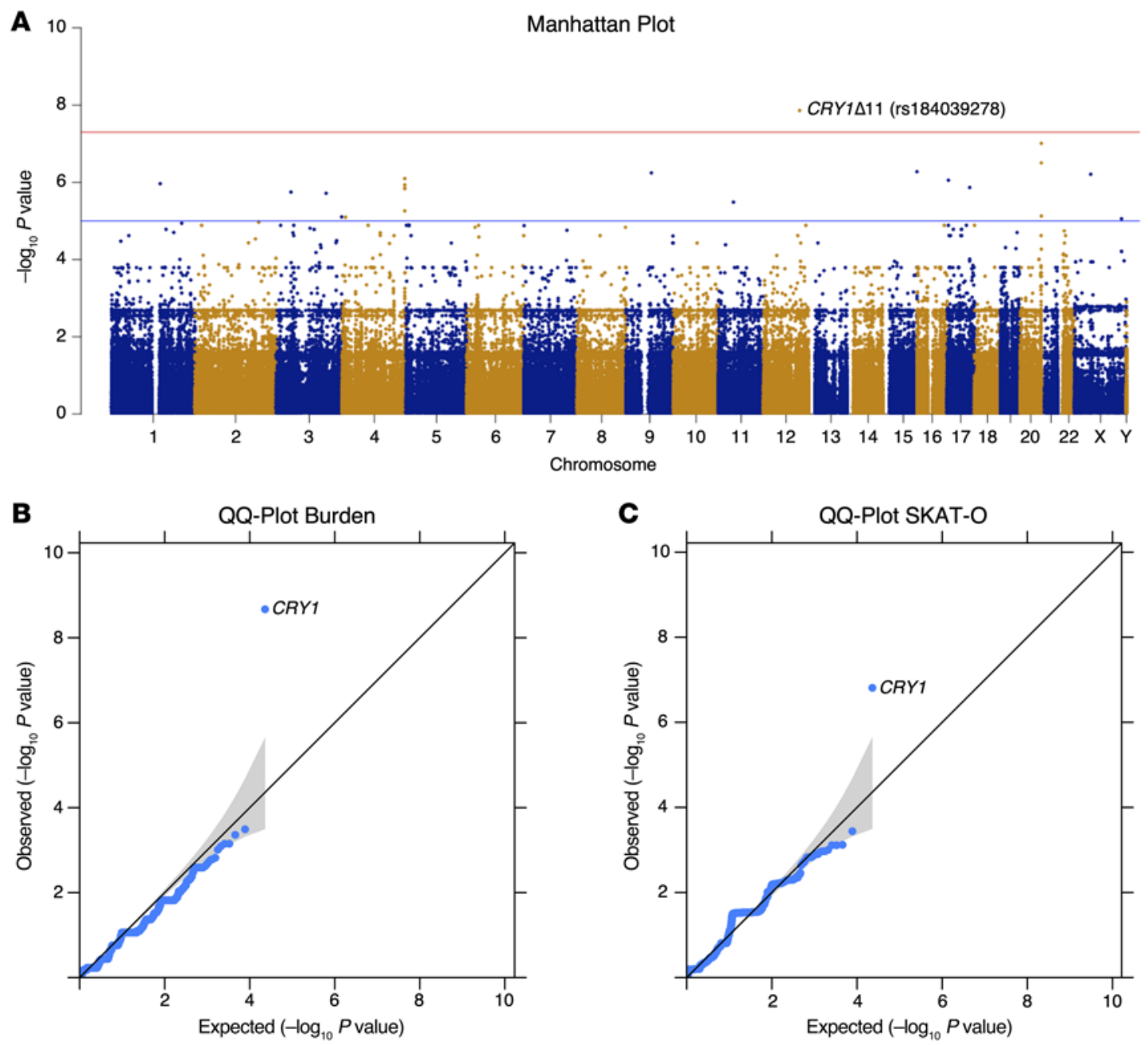

Figure 5. GWAS analyses of ADHD+ versus ADHD' groups. ADHD', affected, $n=78$; $A \mathrm{AHD}^{-}$, unaffected, $n=369$. (A) Manhattan plot for genome-wide association of single nucleotide variants (MAF <0.05). Plots show the $-\log _{10}(P$ value) on the $y$ axis and the chromosomal position of each variant on the $x$ axis. Genes are ranked by uncorrected $P$ values. Red line shows the genome-wide significance cutoff determined by Bonferroni's correction. (B) $Q-Q$ plot showing the observed and expected $P$ values for gene-based burden analysis. (C) Q-Q plot showing the observed and expected $P$ values for gene-based SKAT-O analysis. In the $\mathrm{Q}-\mathrm{Q}$ plots, the expected null distribution (no association) is plotted along the black diagonal with the corresponding $95 \%$ Cls, and the entire distribution of the observed minimum achievable $P$ value-adjusted (MAP-adjusted) $-\log _{10}(P$ value) is plotted in blue.

photolyase homology region (PHR), which leads to the skipping of exon 6 (hence CRY1 6 ). A genotype-phenotype correlation revealed that this variant segregates with ADHD and DSPD in the family (Figure 8, A-C, and Supplemental Table 13). When the phenotype of CRY1 16 carriers was compared with that of CRY1 11 carriers, we noted more severe psychiatric symptoms such as anxiety and oppositional defiant characteristics.

Functional characterization of CRY1 $\triangle 6$. We characterized the functional consequences of the CRY1 $\triangle 6$ variant at the molecular level. The secondary pocket of CRY1 is partially encoded by exon 6 (Figure 8D) and interacts with the Per-Arnt-Sim (PAS-B) domain of CLOCK (36). Docking simulations of modeled CRY1 $\triangle 6$ and WT CRY1 with CLOCK, using HADDOCK (high-ämbiguity-driven protein-protein docking) $(37,38)$, suggested that R256 and F257, encoded by exon 6 of CRY1, are essential for the interaction with CLOCK (Figure 8E). Further analysis indicated that CLOCK does not fit into CRY1 $\triangle 6$ as strongly as it does into WT CRY1 (Supplemental Table 14). We hypothesized that exon 6 of CRY1 could be critical for the interaction with BMAL1 and CLOCK (BMAL1/CLOCK) proteins; therefore, it may be unable to repress BMAL1/CLOCK transactivation. We tested this hypothesis by transfecting human embryonic kidney 293T cells (HEK293T cells) with a Per1::Luc reporter and other appropriate plasmids. Repressor activity of CRY1 $\Delta 6$, which lacks amino acid residues from 229 to 275, was substantially less than that of full-length (FL) WT CRY1 and CRY1 11 (Figure $8 \mathrm{~F})$. Then, we performed coimmunoprecipitation (co-IP) assays to assess the interaction between CRY1 $\triangle 6$ and BMAL1/CLOCK. The CRY1 $\triangle 6$ variant had a severe deficit in its ability to coimmunoprecipitate with the BMAL1/CLOCK heterodimer (Figure 8G), independent of PER2 (Figure 8H). We next used a rescue assay to determine the effect of CRY1 $\Delta 6$ function on the circadian rhythm (39). When expressed under the control of its endogenous promoter and an intronic element, $m \mathrm{Cry} 1 \mathrm{can}$ rescue rhythms in the bioluminescence reporter Per2:Luc (Per2 promoter fused with the luciferase gene) in $\mathrm{Cryy}^{-/-} \mathrm{Cry} 2^{-/}$double-KO (DKO) mouse embryonic fibroblasts (MEFs) (37). Human WT CRY1 and the CRY1D11 variant res- 
A
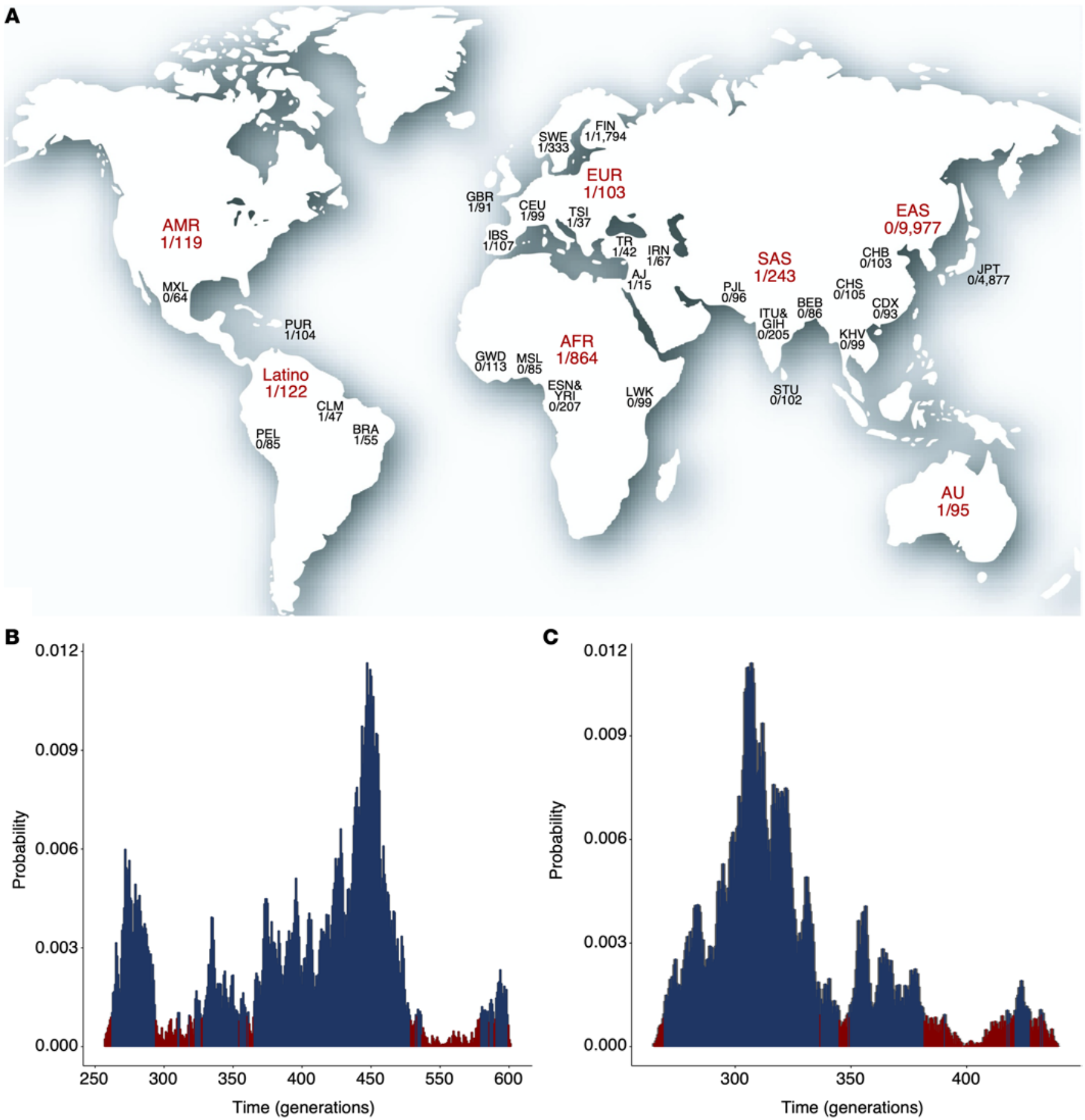

Figure 6. Allele frequency and age distributions of CRY1D11 in different populations. (A) The highest allele frequencies were observed in Ashkenazi Jewish, Italian, Turkish, Brazilian, Iranian, and non-Finnish European populations. The world map is reproduced from 3D Geography. Posterior probability distribution plots depict peaks at (B) 447 generations (TR; 9 carriers and 438 noncarriers; $95 \%$ Cl, 262-548 generations) and (C) 257 generations (CBR, IBS, and CEU; 3 carriers and 294 noncarriers; 95\% Cl, 219-382 generations).

cued the circadian rhythm, but not the CRY1 6 variant (Figure 8I). We also confirmed that the circadian period increased for CRY1 11 by approximately 26 minutes compared with that for WT CRY1, consistent with the previous study (1).

CRY1 stability affects the periodicity of the circadian rhythm $(40,41)$. In order to test the degradation rate of CRY1 $\triangle 6$, we expressed a CRY1 $\triangle 6::$ Luc fusion protein in HEK293T cells and monitored the decay in luminescence as a reporter for protein degradation. Our results indicated that the half-life of CRY1 $\triangle 6(\sim 3$ hours) was significantly higher than that of WT CRY1 ( 1.9 hours) and CRY1 111 ( 2 hours) (Figure 8J). Collectively, these data show that although CRY1 $\triangle 6$ is more stable than WT CRY1, its reduced affinity for BMAL1/CLOCK caused an arrhythmic phenotype when it was expressed in the DKO MEFs. 


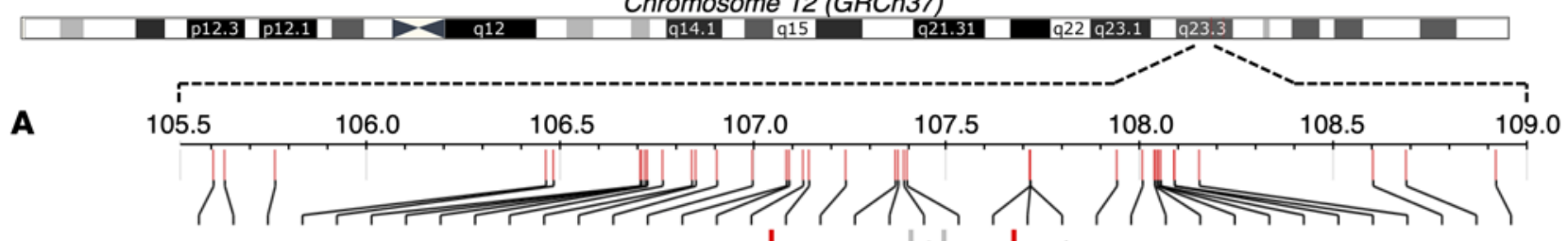

m

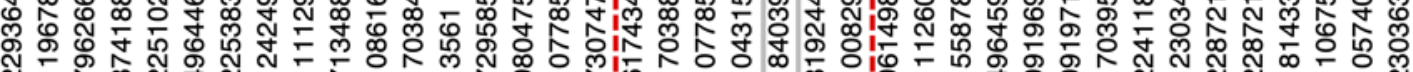

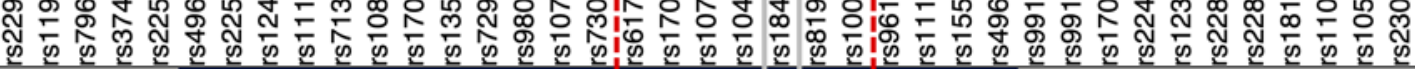

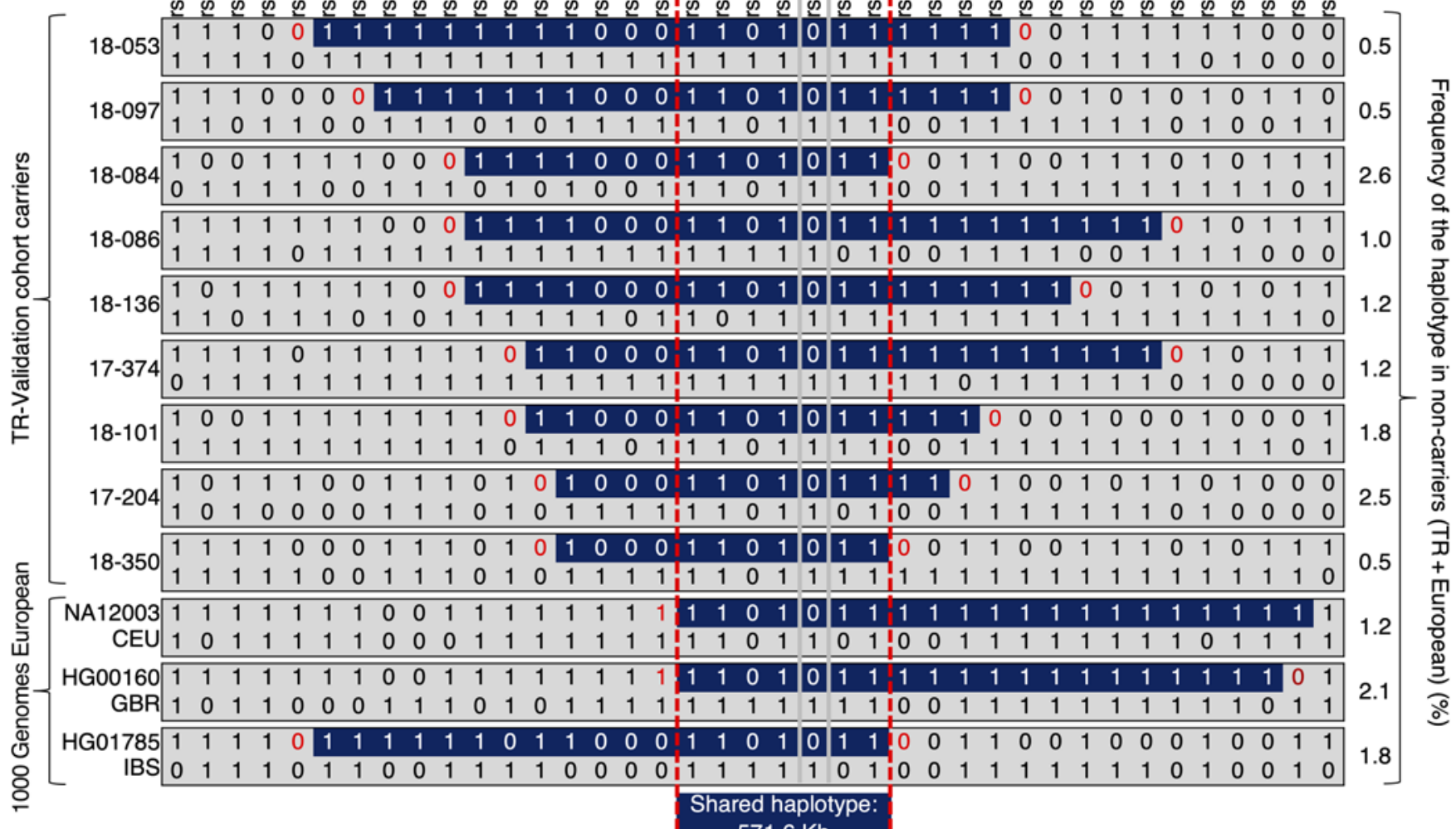

B

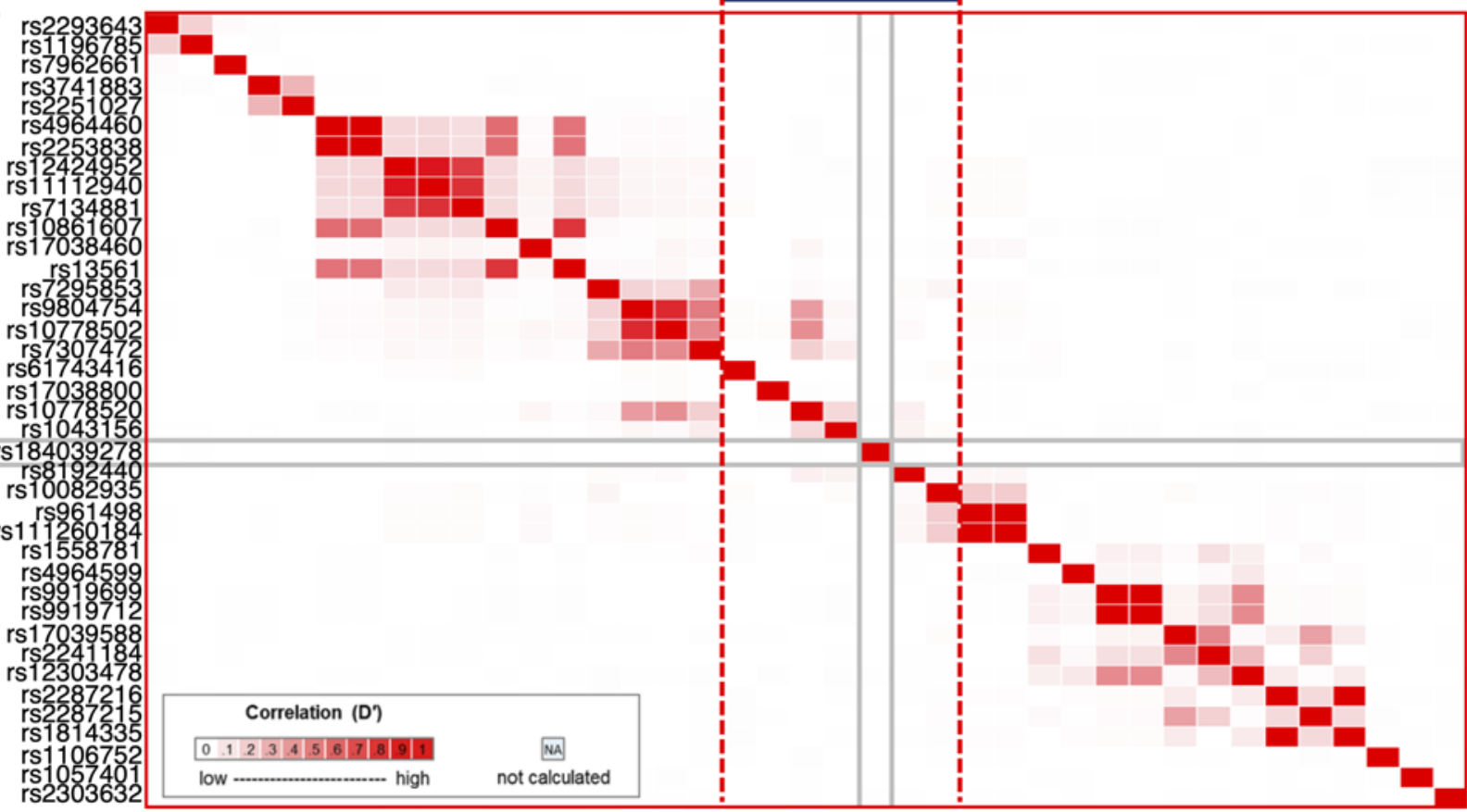


Figure 7. Shared haplotypes for 39 SNPs at 12q23.3 and LDmatrix. (A) Shared haplotypes in the 3-Mb region spanning the $C R Y 1$ gene in carriers of $C R Y 1 \Delta 11(n=12)$ and noncarriers $(n=732)$ from the 447 -individual validation and the 1000 Genomes Project cohorts. Only the haplotypes of the carriers and the frequency of the haplotypes in noncarriers are shown in the figure. All the markers on chromosome 12 were phased using SHAPEIT, version 2.17, but only 39 are represented. (B) Heatmap of pairwise LD statistics for 39 SNP targets that were determined with the LDmatrix module of LDlink software using the 1000 Genomes Project European subpopulation. Pairwise LD values between the SNPs are described by white-red shading: $R^{2}=0$, white; $R^{2}=1$, red.

\section{Discussion}

Our genotype-first analysis identified the first coding variants to our knowledge to be significantly associated with ADHD and indicates an important role for a common variant that alters the circadian rhythm. ADHD is a highly heritable psychiatric disorder that affects nearly $5 \%$ of children and teenagers and $2.5 \%$ of adults globally. ADHD is considered primarily a disorder of impulse control deficit, difficulties in delaying gratification, altered patterns of motivation, and hyperkinesis (42). Interestingly, functional connectivity of brain networks involved in behavior and cognition are implicated in the etiology of ADHD. More specifically, neuroimaging research has revealed functional and maturational abnormalities such as a delay in reaching peak thickness of much of the cerebrum including the prefrontal cortex. Also, delayed maturation and atypical interactions of brain networks, which are involved in the regulation of attentional resources, were shown using functional neuroimaging. In addition, reduced activation of the dopaminergic mesolimbic system was observed in individuals with ADHD (see ref. 42 for a comprehensive review).

Core clock proteins act on nearly $30 \%$ of all genes by binding to the E-box sequence (CAGGTG) in their promoters and hence exhibit daily rhythmicity (43). Clock, Bmal, Per, and Cry genes are expressed broadly throughout the brain, including in several limbic regions responsible for mood regulation and brain reward. Experiments using Drosophila and mice demonstrated that mutations in circadian genes result in depressive or manialike symptoms and affect sensitization to addiction (7). Polymorphisms in CLOCK and PER3 were also suggested to be associated with an increased rate of depressive relapses in patients with bipolar disorder. Therefore, it is conceivable that mutations in circadian genes can interfere with the function of a substantial number of genes that underlie various psychiatric phenotypes. It is tempting to speculate that disturbances in the daily rhythmicity of clock-controlled genes are critical for adaptive plasticity of the brain and optimal functioning of neuronal structures that are important for an ongoing process of assessing the environment, coping with it, and enabling the individual to anticipate and deal with future challenges.

$C R Y$ genes are essential cogs in the core clock machinery. Our characterization of $C R Y 1 \triangle 11$ and $C R Y 1 \triangle 6$ mutations in individuals with combined ADHD and DSPD has uncovered what we believe to be a novel mechanism for a distinct combination of behavioral phenotypes in humans. In addition to ADHD and DSPD, we detected high rates of ADHD comorbidities including depression and smoking behavior in $C R Y 1 \triangle 11$ carriers. Furthermore, we identified significant associations of CRY1 111 with MDD, anxiety, nicotine dependence, and glaucoma. Our results contribute to an unveiling of mechanisms behind the biological overlap of sleep traits with psychiatric traits, as well as support the previously reported findings on $\mathrm{ADHD}$ comorbidities $(10,12)$.

Both the CRY1 111 and $C R Y 1 \Delta 6$ mutations affect the periodicity of the circadian rhythm. Whereas $C R Y 1 \triangle 6$ causes an arrhythmic phenotype, CRY1 $\triangle 11$ lengthens the circadian period by approximately half an hour. We did not observe a significant difference in the severity of ADHD in individuals carrying CRY1 11 or $C R Y 1 \triangle 6$; however, we noted more severe psychiatric symptoms related to anxiety and oppositional defiant disorder in CRY1 $\triangle 6$ carriers. In terms of frequency, $C R Y 1 \triangle 6$ is a private variant, whereas $C R Y 1 \Delta 11$ appears with high frequency in the Eastern Mediterranean, European, and European-derived populations. Allele frequency data and haplotypes of $C R Y 1 \Delta 11$ carriers from different populations indicate that the mutation might have originated in individuals from the Eastern Mediterranean region and expanded to the West into Europe and to the East into Persia, consistent with the Neolithic migration of Anatolian farmers (33).

Multiple studies report lower rates of ADHD in regions with high solar intensity (27). In order to determine whether exposure to sunlight ameliorates ADHD symptoms, we recorded the mean durations of sun exposure in CRY1 mutation carriers. Interestingly, we found that carriers with longer sun exposure durations, especially work-imposed exposure, either had milder phenotypes or were in partial remission. These results suggest that sunlight exerts a protective effect for ADHD symptoms.

\section{Table 1. Association of CRY1 11 with the BioMe BioBank phenotypes}

\begin{tabular}{|c|c|c|c|c|c|c|c|c|}
\hline Phenotype name & No. of cases & Carrier case/control & WT & OR (95\% Cl) & $P$ & MAF & Incidence ( 1 in ...) & ICD-10-CM \\
\hline MDD, single episode & 636 & $37 / 201$ & $599 / 6226$ & $1.91(1.29-2.75)$ & $7.87 \times 10^{-4}$ & 0.03 & 17 & $\begin{array}{c}\text { F32.0, F32.1, F32.2, F32.4, F32.5, } \\
\text { F32.9, F32.89 }\end{array}$ \\
\hline Insomnia & 488 & $28 / 210$ & $460 / 6365$ & $1.84(1.18-2.78)$ & $3.87 \times 10^{-3}$ & 0.03 & 17 & C47.00, F51.01, F51.09 \\
\hline Glaucoma & 54 & $6 / 232$ & $48 / 6777$ & $3.65(1.26-8.66)$ & $7.11 \times 10^{-3}$ & 0.06 & 9 & H40.9 \\
\hline$M D D$, recurrent & 100 & $8 / 230$ & $92 / 6733$ & $2.55(1.05-5.31)$ & $1.28 \times 10^{-2}$ & 0.04 & 13 & $\begin{array}{c}\text { F33.0, F33.1, F33.2, F33.40, F33.41, } \\
\text { F33.42, F33.8, F33.9 }\end{array}$ \\
\hline ADHD & 43 & $3 / 235$ & $40 / 6785$ & $2.17(0.42-6.87)$ & $1.20 \times 10^{-1}$ & 0.03 & 14 & F90.0, F90.1, F90.2, F90.8, F90.9 \\
\hline
\end{tabular}


A

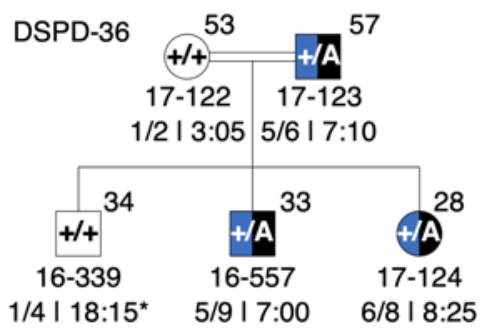

B

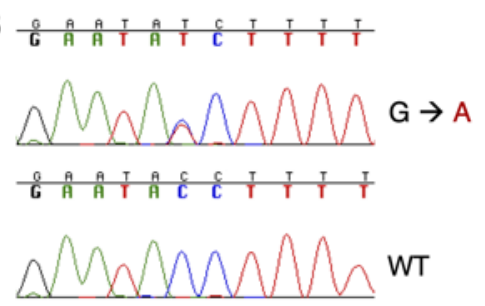

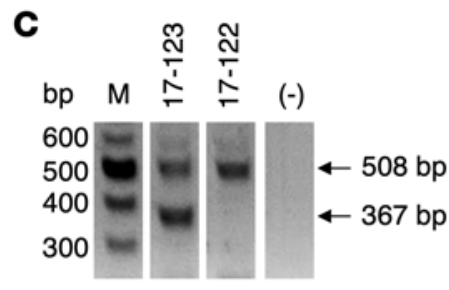

D

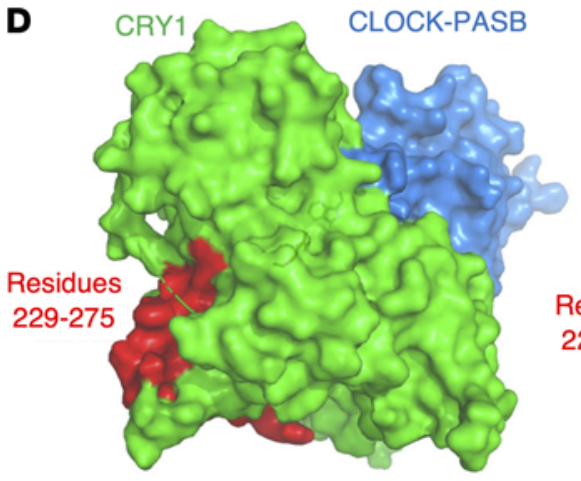

G

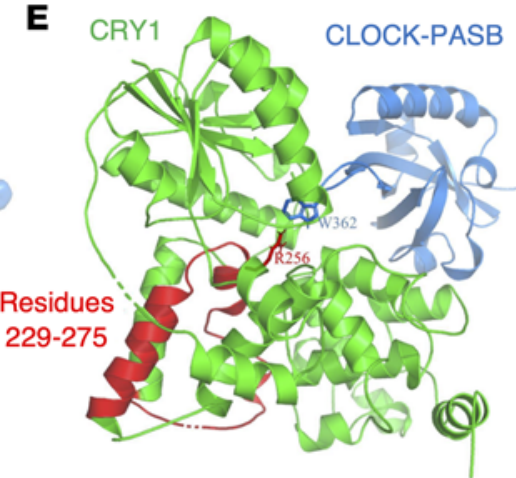

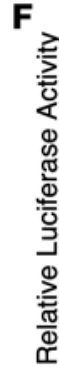

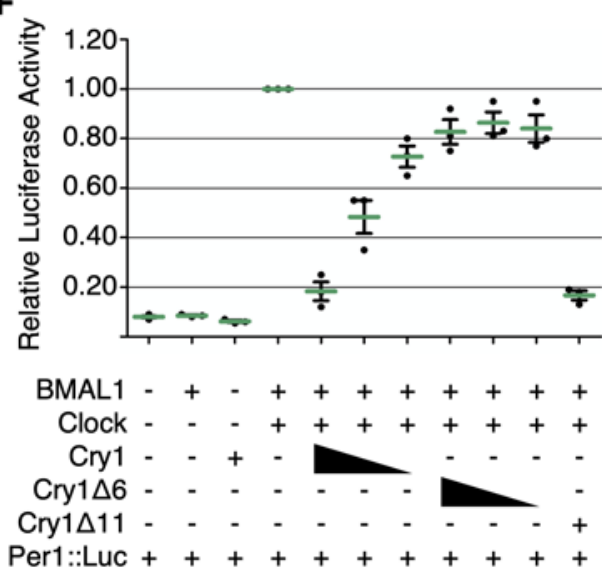

J

I
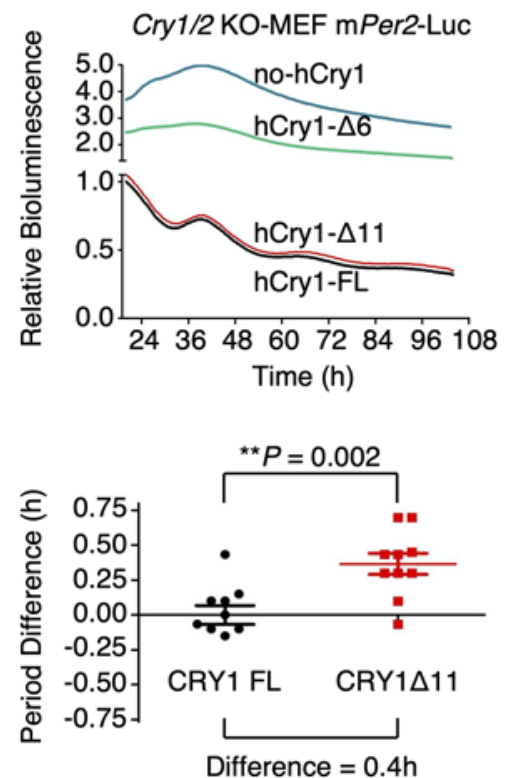
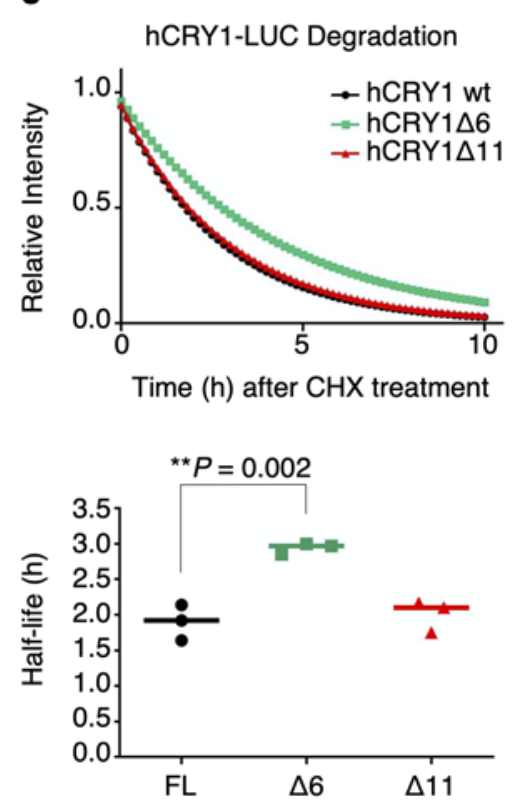

Figure 8. Phenotype-genotype characterization of family DSPD-36 and functional characterization of CRY1 $\Delta 6$. (A-C) Phenotype-genotype characterization of family DSPD-36. (A) The family DSPD-36 was assessed as described in the legend to Figure 3. (B and C) c.825+1G>A causes skipping of 141-bp exon 6 , leading to an in-frame deletion of 47 residues in the middle of the PHR and clock-binding domains of the CRY1 protein. M, DNA marker. (D-J) Functional characterization of CRY1 $\triangle 6$. (D) Docking analysis of CRY1 and the CLOCK PAS-B domain. (E) The critical amino acid residue of CRY1 (R256) interacts with CLOCK PAS-B (W362). (F) Analysis of the effect of FL WT CRY1, CRY1 6 6, and CRY1 11 on CLOCK/BMAL1-driven transcription with the Per1:Luc assay. An E-box-driven luciferase reporter plasmid Per1:Luc was coexpressed in HEK293T cells along with plasmids consisting of CLOCK and BMAL1 cDNAs and decreasing amounts of WT CRY1 or CRY1 $\triangle 6$. Data represent the mean induction of bioluminescence over basal levels from duplicate transfections 48 hours after transfection. Error bars represent the SD from at least 3 biological experiments. Co-IP assay with (C) CLOCK, BMAL1, (H) PER2, CLOCK, BMAL1, and human CRY1s (hCRY1). Blots in $\mathbf{G}$ and $\mathbf{H}$ are representative of at least 3 independent experiments. (I) Rescue assays were performed with human CRY1s with at least 4 biological replicates. Samples were normalized to the initial luminescence signal. The graph below indicates the period differences, with the whiskers representing the mean \pm SEM values. $n=5$ per group. Data were pooled from 2 independent experiments. ${ }^{*} P=0.002$, by unpaired $t$ test. mPer2-Luc, mouse Per2-Luc. (J) Degradation assay with human CRY1::Luc and its variants. The graph below was generated by fitting a 1-phase decay curve to the data, which indicate the half-life, with the horizontal line representing the mean. $n=3$ per condition, pooled from 3 independent experiments. ${ }^{*} P=0.002$, by unpaired $t$ test. 
In conclusion, we describe a monogenic form of combined ADHD and DSPD frequently accompanied by a history of depression due to pathogenic CRY1 mutations. Furthermore, our findings provide a mechanistic explanation for the development of these behavioral phenotypes by linking a common and causal genetic variant with a compromised circadian period due to disturbances in the negative feedback loop of the core molecular clock. Although these observations are consistent with a substantial epidemiological comorbidity of psychiatric disorders, we believe they provide a novel perspective on their genomic architecture. Psychiatric disorders are characterized by a polygenic nature with many genetic loci contributing to risk (13). However, in the case of CRY1, mutations at a single locus could lead to what may be one of the most common autosomal dominant disorders. Therefore, $C R Y 1 \triangle 11$ has significant potential in diagnostic testing (44) and presents a target for therapeutic intervention. Reverse phenotyping of individuals and families with damaging mutations in core clock genes and genotyping for circadian rhythmicity in well-characterized cohorts of psychiatric disorders could pave the way to dissect the constitutional determinants of a distinct group of circadian psychiatric phenotypes that we propose to designate as circiatric disorders.

\section{Methods}

Patient evaluations and genetic material. Clinical information included medical history, a physical examination, a psychiatric evaluation, pedigree drawings, a complete blood count, blood and urine biochemistry analysis, and height and weight measurements for BMI determination. Genomic DNA was extracted from blood cells using standard procedures and the NucleoSpin Blood L Kit (Macherey-Nagel). For those families living outside of Ankara, several of the investigators traveled to the families' hometowns in Konya (DSPD-1), Urfa (DSPD4), and Kayseri (DSPD-6) to perform the clinical evaluations. Families in Italy were evaluated at Siena University. All participants completed the adult ASRS questionnaire (22), developed by the WHO to measure symptoms of ADHD, and the MCTQ (21). The psychiatric analysis began from the childhood period, and paid special attention to establishment of trust to minimize the drive to give appropriate rather than candid answers. Clarity of communication was equally important to make sure that correct words were chosen in the expression of emotions by each subject. Special attention was paid to dissect whether symptoms were secondary to another psychiatric disorder. Several of the investigators reviewed the ASRS questionnaires and the MCTQs completed by each participant. Clinical data on the family members (DSM-5 ADHD and ASRS scores, ADHD severity, demographics, ADHD symptoms, and sleep behavior) are presented in Supplemental Tables 1-3. ADHD diagnosis according to the DSM-5 requires 6 symptoms for children younger than 17 years of age and 5 or more symptoms for older adolescents and adults. Phenotype components in the families included excessive inattention and/or hyperactivity and impulsivity as well as executive dysfunction, lack of emotional self-control, and motivation frequently present with characteristics of oppositional defiance. The current severity of ADHD was specified as mild (few if any symptoms, which result in only minor functional impairments); moderate (functional impairments or symptoms between mild and severe); and severe (presence of symptoms that result in marked impairments in social or occupational functioning).
In Figure 3, ADHD is represented with blue and DSPD with black colors. Individuals for whom a definitive ADHD diagnosis was made were further classified as combined (when all 3 core features of inattention, hyperactivity, and impulsivity were present); predominantly inattentive (diagnosed if $\geq 5$ symptoms of inattention but $<5 / 6$ symptoms of hyperactivity/impulsivity had persisted for $\geq 6$ months); and predominantly hyperactive/impulsive (diagnosed if $\geq 5 / 6$ symptoms of hyperactivity/impulsivity but $<5 / 6$ symptoms of inattention had persisted for $\geq 6$ months). Supplemental Table 2 provides data on the DSM-5 symptoms of inattention (questions 1-9), DSM-5 symptoms of hyperactivity and impulsivity (questions 10-18), and ASRS scores for the 14 families and individual family members. Questions 1-4 of Part A and questions 7-11 of Part B are for inattention, and questions 5 and 6 of Part A and questions 12-18 of Part B are for hyperactivity/ impulsivity evaluation. Sleep behavior was independently assessed by several of the investigators through a sleep interview, which included sleep and ChronoType questionnaires. As previously reported (1), for the families DSPD-1, -4, -6, -7, -9, and -14, DSPD is part of the behavioral phenotype and is also present in families DSPD-2, -31, -34, -51, $-52,-53,-58$, and -59 (Supplemental Table 3) reported in the current study. Note that data on additional individuals from families DSPD-1, $-4,-6$, and -9 are now presented in Supplemental Table 3. Nine families (DSPD-1, -2, -4, -14, -6, -9, -51, -52, and -31) are consanguineous or endogamous (Figure 3) and are from different cities or towns located in Anatolia. An important observation emerging from DSPD-4 and DSPD-52 is that there is no marked phenotypic difference for DSPD or ADHD between the homozygotic (16-006, 16-008, 16-018, 16-042, 16-049, 17-281) and heterozygotic (16-014, 16-015, 16-016, 16-027, 16-043, 16-052, 17-011, 17-292) individuals.

CRY1 c.1657+3A>C amplification and genotyping. CRY1 c. $1657+3 \mathrm{~A}>\mathrm{C}$ genotype status was determined by amplifying genomic DNA using hCryli10F (5'-GTCAACACTTCTGTGAGCCT-3') and hCryli12R (5'-CAGATGCATGTCTCTTGACC-3') and restriction digestion analysis (1). The PCR yielded a 623-bp product of the genomic locus containing exon 11 and was digested with Hpy188I (+ allele: no cut, variant c.1657+3A>C: 276 bp + 347 bp; Supplemental Figure 2).

Whole-exome sequencing. Whole-exome sequencing (WES) was performed on genomic DNA from the 447-individual validation cohort at the YCGA (Supplemental Table 15). Exome capture was done using the xGen Exome Research Panel (version 1.0) Capture Kit (Integrated DNA Technologies [IDT]) according to the manufacturer's protocol. Samples were sequenced on the HiSeq 4000 platform (Illumina) with 100-bp paired-end reads. WES data and associated sample information described in Supplemental Table 6 have been deposited in the NCBI's dbGAP repository (accession ID: BioProject PRJNA624188; https://www.ncbi.nlm.nih.gov/Traces/study/?acc=PRJNA624188). Base calling, read filtering, and demultiplexing were performed with the standard Illumina processing pipeline. The read pairs were mapped to the human genome build GRCh37 with the Burrows-Wheeler Aligner (BWA), version 0.7.17, with default settings (45). Aligned duplicate reads were marked using Mark Duplicates in Picard tools. GATK, version 3.7 (Genome Analysis Toolkit [GATK]), was used for base quality score recalibration (BQSR) and local realignment around indels to refine alignment artifacts around putative insertions or deletions (46). Variant discovery was performed in 2 steps, beginning with variant calling with GATK HaplotypeCaller (https://gatk.broadinstitute.org/ 
hc/en-us/articles/360037225632-HaplotypeCaller) followed by joint genotyping using GATK GenotypeGVCFs (https://gatk.broadinstitute. org/hc/en-us/articles/360037057852-GenotypeGVCFs). Variants with a Phred quality score below 30 were removed. The resulting variant call set was refined using variant quality score recalibration (VQSR) as implemented in GATK VariantRecalibrator (https://gatk.broadinstitute.org/hc/en-us/articles/360036510892-VariantRecalibrator). Variant recalibration was applied by the GATK ApplyRecalibration walker using a tranche sensitivity of $99.5 \%$ for SNPs and $99.0 \%$ for indels. VQSR was used to define low-quality variants for downstream processing (Supplemental Table 16).

Variants were trimmed and left-aligned around indels, and multiallelics were split using GATK, version 3.7. A total of 886,935 variants were obtained. Sample-based quality control was carried out using PLINK, version 1.9, software (47). No low-quality samples with more than $10 \%$ missing genotypes were identified. Sex verification and kinship analysis were performed using KING software (48). No related individuals were detected (degree $=2$, kinship coefficient $\geq 0.0625$ ) in the validation cohort (Supplemental Figure 1, A and B). To determine outliers of the population, a principal component (PC) analysis was conducted on a subset of the common biallelic variants $(n=43,557)$ pruned for linkage disequilibrium (LD) using the PLINK, version 1.9, "indep-pairwise" command (window = 50 SNPs, step size $=5$ SNPs, maximum $r 2=0.5$ ). The first 10 PCs were calculated using the "smartpca" module of the EIGENSTRAT method (EIGENSOFT package) (49), and no outlier samples were observed (Supplemental Figure 1C).

Variant annotation and prioritization. Variants in protein coding genes were identified by SnpEff, version 4.4 (50), which uses the ENSEMBL, version 87, gene models to determine variant functional region and impact on the assigned gene. Variants were annotated using ANNOVAR (version 2019Oct24) (51). Variants were subsequently filtered out on the basis of quality control scores, MAFs, deleteriousness/ functional impact, and variants at low-complexity regions.

Briefly, common variants defined by a MAF of more than $0.1 \%$ in GnomAD, version 2.1.1 (31), Kaviar (52), 1000 Genomes Project (32), or ESP6500 (53), and an in-house Turkish unrelated control database of 2628 whole-exome and 773 whole-genome data sets were excluded from the analysis. The potential impact of missense variants was predicted using MetaSVM (54) and Combined Annotation-Dependent Depletion (CADD) (55) tools, and that of splice site variants located \pm 3 bp of exon-intron junctions was predicted using dbscSNV-ADA/-RF (56) and Spidex scores (ref. 57 and Supplemental Table 16).

Candidate gene prioritization approach. We performed analyses of associations between ADHD and rare deleterious mutations using 31,432 variants on 11,528 genes. Single variant-based analysis was carried out using PLINK, version 1.9. Small-sample adjustments and rare variant weights were used for gene-based analysis with SKAT-O and the burden test with Bonferroni's multiple testing correction (30). The results of the single-variant association test and gene-based statistical analyses were used to create Manhattan and quantile-quantile (Q-Q) plots, respectively (Figure 5, A and B). Although small-sample adjustments were applied, the Q-Q plots still had a slightly anticonservative pattern.

Other core clock genes. The presence of additional polymorphisms was not unexpected, given the large degree of variation commonly found in human clock genes $(58,59)$. In addition to the CRY1 c. $1657+3 \mathrm{~A}>\mathrm{C}$ variant, other coding, rare, and deleterious vari- ations of core clock genes (CRY1, CRY2, PER1, PER2, PER3, ARNTL, $C L O C K$, and CSNK1D) and additional candidate clock genes (CSNK1E, ARNTL2, FBXL3, FBXL21, BHLHE4O, BHLHE41, NR1D1, and RORA) were identified, but none of the genes other than $C R Y 1$ were statistically significant for the association with the phenotypically distinguished groups tested (Supplemental Tables 12 and 17).

Haplotype analysis. We inferred haplotypes from chromosome 12 of the 447-individual validation cohort and European populations from 1000 Genomes Project, Phase 3 using phased SNPs by SHAPEIT, version 2.17 (https://mathgen.stats.ox.ac.uk/genetics_software/ shapeit/shapeit.html). The same SNPs were used to calculate the linkage disequilibrium across ancestral populations using LDlink (https:// ldlink.nci.nih.gov/?tab=home) and to estimate age using DMLE+. SHAPEIT uses a set of genotypes and a genetic map and produces a set of estimated haplotypes 60). We investigated the 1000 Genomes Project, Phase 3 haplotype data and noted 3 CRY1 11 carriers in the European populations of Utah residents with Northern and Western European ancestry (CEU), the British in England and Scotland (GBR), and the Iberian population in Spain (IBS). All 594 European and 894 Turkish (TR) haplotypes were combined. Thirty-nine phased, biallelic, informative SNPs (MAF >0.05) in the $3-\mathrm{Mb}$ region spanning CRY1 $\triangle 11$ were evaluated, and 12 different haplotypes in carriers were identified. These haplotypes share a $571.6 \mathrm{~kb}$ common segment, which involves CASC18, NUAK1, TCP11L2, POLR3B, RFX4, MTERF2, TMEM263, $C R Y 1, B T B D 11, P W P 1, P R D M 4$, WSCD2, and CMKLR1. The frequencies of 12 haplotypes were in the range of $0.5 \%$ to $2.6 \%$ in 732 noncarriers (Figure 7A).

To assess LD across ancestral populations, the LDmatrix module of LDlink (61) was used, and an interactive heatmap matrix of pairwise linkage disequilibrium using 39 SNPs was created (Figure 7B). The 1000 Genomes Project, Phase 3 haplotype data on populations from CEU, GBR, IBS, and Tuscany in Italy (TSI) were extracted.

Age estimation of CRY1 11 in Turkish and European populations. DMLE+, version 2.3 (62), was used to estimate the age of the CRY1 11 mutation, with the recommended burn-in and sampling intervals and a variety of parameter ranges. We used the haplotypes generated from 38 phased SNPs spanning $3 \mathrm{Mb}$ around the $C R Y 1 \Delta 11$ mutation in unrelated individuals. The population growth rate (r) was estimated (e) using the equation: $\mathrm{T}_{1}=\mathrm{T}_{0} \mathrm{e}^{(\mathrm{gr})}$, in which $\mathrm{T}_{1}$ is the estimated size of the current population, $\mathrm{T}_{0}$ is the estimated size of the ancestral population, and $g$ is the number of generations between these 2 time points (63). The growth rate of the Turkish population was estimated as $0.009\left(\mathrm{~T}_{1}\right.$ $=81.81$ million, $\mathrm{T}_{0}=12$ million $[200 \mathrm{BCE}]$ and $\left.\mathrm{g}=88.7\right)(64)$, and the growth rate of the European (GBR, IBS, and CEU) populations in the 1000 Genomes Project was estimated as $0.016\left(\mathrm{~T}_{1}=116.26\right.$ million, $\mathrm{T}_{0}$ $=20.75$ million on $1 \mathrm{CE}$, and $\mathrm{g}=80.7)(65)$. A 25 -year intergeneration interval was used for calculations. The "proportion of disease-bearing chromosomes sampled" was estimated as $3.11 \times 10^{-6}$ for the Turkish cohort and $5.47 \times 10^{-6}$ for the European cohort, using the population sizes $\left(\mathrm{T}_{1}\right)$ and carrier frequencies (1 of 42 and 1 of 100). The mutation density depicted a peak at 447 generations (95\% credible set $=262$ 548 ) and 257 generations ( $95 \%$ credible set $=219-382$ ) for the Turkish and the European populations, respectively.

PheWAS of CRY1 11 in the BioMe BioBank. PheWAS analyses for $C R Y 1 \triangle 11$ were performed on the basis of electronic medical recordlinked phenotypes (ICD-10-CM codes) in the BioMe BioBank of the Institute for Personalized Medicine at the Icahn School of Medicine at 
Mount Sinai. The phenotype information and CRY1 11 status of 9438 unrelated adult Europeans were analyzed. The CRY1 11 phenotype association was tested independently using Fisher's exact test.

Reverse transcription PCR analysis of CRY1 $m R N A$. Fresh venous blood samples were collected into PAXgene Blood RNA tubes (PreAnalytiX), and total RNA was isolated from subjects 17-122 and 17-123 using the QIAamp RNA Blood Mini Kit with on-column DNase digestion (QIAGEN). Equal amounts of total RNA were used for first-strand cDNA synthesis using the RevertAid First-Strand cDNA Synthesis Kit with Oligo(dT) ${ }_{18}$ priming followed by RNase $\mathrm{H}$ digestion (Thermo Fisher Scientific). The resulting coding change of CRY1 c.825+1G>A was tested by amplifying the part of the cDNAs between exons 5 and 8 using CR508F (5'-GGAGAAACTGAAGCACTTACTC-3') and CR508R (5'-CAAATACCTTCATTCCTTCTTCCC- $\left.3^{\prime}\right)$. The PCR yielded a FL 508-bp product in the WT individual and an additional 367-bp product in the heterozygous proband (Figure 8C).

Human CRY1 cloning and mutagenesis. The WT coding sequence for CRY1 was obtained from a human cDNA sample designated as 17-125. Two microliters of this cDNA sample was amplified via touchdown PCR using Phusion polymerase (Thermo Fisher Scientific) and the primers provided in the Supplemental Table 18. Amplified fragments were initially cloned to pJET1.2/blunt vector (Thermo Fisher Scientific) using the CloneJET PCR Cloning Kit (Thermo Fisher Scientific) for individual clones destined for pcDNA4A and pMU2. Sequence verification of amplified inserts was performed via automated Sanger sequencing (Macrogen) to confirm the absence of mutation(s) possibly introduced during the PCR amplification. Sequence-verified inserts were subcloned to their respective vectors (pMU2 and pcDNA4/Myc-His A) via restriction/ligation. Mutagenesis was performed for both CRY1 integrated constructs via Phusion-based, site-directed mutagenesis using the oligonucleotide primers listed in Supplemental Table 19. Sequence verification was repeated as described above. PCR reactions for each construct were prepared in $50 \mu \mathrm{L}$ of total volume using the reaction conditions in Supplemental Table 20.

To clone CRY1 into pMU2 and pcDNA4/Myc-His A, flanking XbaI and NotI sites were added to the primers. For the pcDNA4/Myc-His A construct, a stop codon was removed, and 2 extra nucleotides were added to include the His tag present in the plasmid.

Touchdown PCR was performed on a T100 Thermal Cycler (BioRad). PCR reactions were set up on ice and transferred to a preheated thermocycler. The cycling conditions for the touchdown PCR reaction are described in Supplemental Table 21.

The sizes of the PCR products were verified by agarose gel electrophoresis, and the band corresponding to CRY1 was excised and purified using a NucleoSpin PCR and Gel Purification Kit (Macherey Nagel). The purified CRY1 fragment was then ligated to an empty pJET1.2/ blunt vector using a CloneJET PCR Cloning Kit (Thermo Fisher Scientific) following the manufacturer's protocol. Ligation reactions $(5 \mu \mathrm{L})$ were transformed to DH5 $\alpha$ cells, and transformed cells were spread on Luria-Bertani (LB) broth agar plates supplemented with $100 \mu \mathrm{g} / \mathrm{mL}$ ampicillin and then incubated overnight at $37^{\circ} \mathrm{C}$. Colonies from plates were selected the next day and grown in $2 \mathrm{~mL}$ liquid LB, and plasmids were purified using a plasmid purification kit (Macherey Nagel). Plasmids were then digested with the appropriate restriction endonucleases to confirm the presence of the insert with gel electrophoresis and plasmids. The plasmids were then sequenced to confirm the absences of mutation(s) of the CRY1 gene via automated Sanger sequencing
(Macrogen). Next, plasmids were double digested with XbaI/NotI for pMU2 inserts and EcoRV/NotI for pcDNA4/Myc-His A inserts. pMU2 and pcDNA4/Myc-His A were also double-digested with XbaI/ NotI and EcoRV/NotI, respectively. Digested destination vectors were treated with FastAP (Thermo Fisher Scientific) to limit self-annealing. Both inserts and vectors were gel purified and ligated using T4 DNA ligase (Thermo Fisher Scientific) to generate pMU2-hCRY1 and pcDNA4A-hCRY1 plasmids and then transformed into DH5 $\alpha$ cells. pMU2CRY1-transformed cells were plated onto LB agar supplemented with $34 \mu \mathrm{g} / \mathrm{mL}$ chloramphenicol, and pcDNA4A-CRY1-transformed cells were plated onto LB agar supplemented with $100 \mu \mathrm{g} / \mathrm{mL}$ ampicillin and incubated overnight at $37^{\circ} \mathrm{C}$. The presence of the human CRY1 cDNAs in these vectors was verified with gel electrophoresis using the appropriate restriction endonucleases.

The deletion of exons 6 and 11 from human CRY1 cDNA was performed with a PCR-based strategy using Phusion Polymerase and asymmetric oligonucleotides incorporating 20-nt homology designed to incorporate missense mutations and deletions on pMU2CRY1 and pcDNA4A-CRY1 constructs (the conditions and forward/ reverse primers for PCR mutagenesis are listed in the Supplemental Tables 22 and 23).

Phusion-based mutagenesis PCR reactions were performed on a T100 Thermocycler (Bio-Rad). PCR reactions were set up on ice and transferred to a preheated thermocycler. The cycling conditions for the mutagenesis PCR reaction are provided in Supplemental Table 23.

After mutagenesis with PCR, the reactions were treated with DpnI at $37^{\circ} \mathrm{C}$ for 3 hours to eliminate parental template plasmid DNA and then transformed into DH5 $\alpha$ cells. pMU2-CRY1-transformed cells were plated onto LB agar supplemented with $34 \mu \mathrm{g} / \mathrm{mL}$ chloramphenicol and pcDNA4A-CRY1-transformed cells were plated onto LB-agar supplemented with $100 \mu \mathrm{g} / \mathrm{mL}$ ampicillin and incubated overnight at $37^{\circ} \mathrm{C}$. The presence of mutations was verified with automated Sanger sequencing (Macrogen).

Real-time bioluminescence rescue assay. Cry $1^{-/-} \mathrm{Cry}^{2--}$ MEFs (CRYDKO MEFs) $\left(3 \times 10^{5}\right)$ were seeded in $35-\mathrm{mm}$ clear tissue culture plates. Cells were transfected with 4000 ng pGL3-Per2-Luc (luciferase reporter) and 150 ng CRY1 plasmid [pMU2-P(CRY1)-(intron 336)]designed to rescue the circadian rhythm using FuGENE 6 Transfection Reagent (Promega) according to the manufacturer's protocols. The total DNA amount was equalized to $4150 \mathrm{ng}$ with pSport6 plasmid if only the reporter plasmid was transfected. Seventy-two hours after transfection, cells were synchronized with $0.1 \mu \mathrm{M}$ dexamethasone for 2 hours. Medium was replaced with bioluminescence recording medium (1\% DMEM powder [w/v], 0.035\% sodium bicarbonate, $0.35 \% \mathrm{D}[+]$ glucose powder, $1 \% \mathrm{~mL} 1 \mathrm{M}$ HEPES buffer, $0.25 \%$ penicillin/streptomycin, $5 \%$ FBS), in which luciferin was freshly supplemented ( $0.1 \mathrm{mM}$ final concentration). Plates were sealed with vacuum grease and placed into the LumiCycle (Actimetrics). Bioluminescence monitoring was performed for 70 seconds every 10 minutes for 7 days via photomultiplier tubes. Luminescence values were recorded and processed using LumiCycle Analysis software. The first 20 hours of data were discarded from the analysis due to transient luminescence upon medium change. Period and amplitude values were obtained using damped sine wave based on the running average option for each sample.

CRY1-mediated repression of BMAL1-CLOCK transcription activity. Low-passage-number HEK293T cells $\left(5 \times 10^{6}\right)$ were seeded onto a $10-\mathrm{cm}$ plate containing $10 \mathrm{~mL}$ DMEM (Gibco, Thermo Fisher Scientific) supple- 
mented with $10 \%$ heat-inactivated FBS (Gibco, Thermo Fisher Scientific), $1 \times$ penicillin/streptomycin (Gibco, Thermo Fisher Scientific), and $4 \mathrm{mM}$ L-glutamine (Gibco, Thermo Fisher Scientific) (denoted as $1 \times$ DMEM). Cells were incubated overnight at $37^{\circ} \mathrm{C}$ in $5 \% \mathrm{CO}_{2}$ until they reached $70 \%-80 \%$ confluence. Cells were washed with $5 \mathrm{~mL} 1 \times \mathrm{PBS}$, trypsinized, and resuspended in DMEM supplemented with 20\% heat-inactivated FBS, $2 \times$ penicillin/streptomycin, and $8 \mathrm{mM} \mathrm{L-glutamine} \mathrm{(denoted} \mathrm{as} 2 \times$ DMEM), such that the total concentration was $8 \times 10^{5}$ cells $/ \mathrm{mL}$. Diluted HEK293T cells were distributed to white Costar 96-well culture plates (50 $\mu \mathrm{L} /$ well), rendering the cell concentration $4 \times 10^{4}$ cells/well.

For each well, a mixture of $50 \mathrm{ng}$ pSport6-BMAL1, 125 ng pSport6CLOCK, 50 ng pGL3-Per1::Luc, 1 ng pRL-TK, 4 ng pcDNA4A-CRY1, and 120 ng empty pSport6 was prepared in DMEM (without FBS or antibiotics). For a positive control, the mixture was supplemented with $4 \mathrm{ng}$ empty pcDNA4/Myc-His A. This mixture was supplemented with 0.9 $\mu \mathrm{L}, 22-\mathrm{kDa}$ linear polyethylenimine (PEI) (Polysciences), vortexed briefly and incubated at room temperature for 20 minutes. Fifty microliters of the mixture was added on top of each well in triplicate. The plates were incubated for 24 hours at $37^{\circ} \mathrm{C}$ in $5 \% \mathrm{CO}_{2}$. Firefly luciferase and Renilla luciferase expression was determined using the Dual-Glo Luciferase Assay System (Promega) according to the manufacturer's protocol.

CRY1-Luc degradation assay. Low-passage-number HEK293T cells $\left(5 \times 10^{6}\right)$ were seeded onto a $10-\mathrm{cm}$ plate containing $10 \mathrm{~mL} 1 \times$ DMEM. Cells were incubated overnight at $37^{\circ} \mathrm{C}$ in $5 \% \mathrm{CO}_{2}$ until $70 \%-80 \%$ confluence. Forty nanograms of the expression vector (CRY1-Luc plasmid) was reverse-transfected into $4 \times 10^{4}$ HEK293T cells on a white 96-well plate with a flat-bottomed via PEI transfection reagent. Cells were treated with luciferin (0.4 mM final) and HEPES (10 mM final and $\mathrm{pH}=7.2)$ after 48 hours of transfection for 2 hours. Cycloheximide (CHX) $(20 \mu \mathrm{g} / \mathrm{mL}$ final) was added to wells to halt the protein synthesis. The plate was sealed with optically clear film. Luminescence readings were collected via Synergy $\mathrm{H} 1$ reader every 10 minutes at $32^{\circ} \mathrm{C}$ for 24 hours. The protein half-life was calculated using 1-phase exponential decay fitting (GraphPad Prism 5, GraphPad Software).

Immunoprecipitation. HEK293T cells $\left(4 \times 10^{5}\right.$ per well) were seeded onto 6-well tissue plates 24 hours before the transfection. Cells were transfected via PEI with CRY1-His-Myc, CRY1- $\triangle 11-\mathrm{His}-\mathrm{Myc}$, or CRY1$\triangle 6$-His-Myc in pcDNA4-A or empty sport6 with Flag-CMV-BMAL1, or Flag-CMV-CLOCK for IP with BMAL1, CLOCK, and CRY. The FlagPER2-CMV plasmid was also transfected along with BMAL1, CLOCK, and $C R Y$ plasmids to immunoprecipitate 4 clock proteins. For negative control BMAL1, CLOCK or BMAL1, CLOCK, and PER2 were transfected with empty sport6 plasmid instead of $C R Y$ via PEI transfection reagent. Twenty-four hours after transfection, the cells were harvested via icecold PBS. After centrifugation, the pellets were lysed in $300 \mu \mathrm{L}$ passive lysis buffer (PLB) (15 mM HEPES, $300 \mathrm{mM} \mathrm{NaCl}, 5 \mathrm{mM} \mathrm{NaF}, 1 \%$ NP4O supplemented with fresh protease inhibitor) for 20 minutes on ice. To get rid of cell debris, the samples were centrifuged for 15 minutes at $13,000 \times g$ at $4^{\circ} \mathrm{C}$. Ten percent of the supernatant was saved as input. Ni-NTA agarose resin $(15 \mu \mathrm{L})$ (QIAGEN) per sample was equilibrated by washing 2 times with TBS-300 ( $15 \mathrm{mM}$ Tris, $300 \mathrm{mM} \mathrm{NaCl}$ ) supplemented with $25 \mathrm{mM}$ imidazole and 2 times with PLB. The remaining supernatant was added onto the equilibrated resins with $25 \mathrm{mM}$ imidazole. The cell lysates and resins were incubated for 1.5 hours to pull down CRYs. The resins were washed 4 times with TBS-300 $(300 \mu \mathrm{L})$ with 25 $\mathrm{mM}$ imidazole. Proteins were isolated from resins by boiling in Laemmli buffer (31.5 mM Tris-HCl, pH 6.8 buffer 10\% glycerol, 1\% SDS, 0.005\% bromophenol blue, and freshly added $5 \% \beta$-mercaptoethanol).

Anti-Flag antibody (MilliporeSigma, A9469) was used to detect BMAL1, CLOCK, and PER2. Blots were stripped (Advansta Strip-It Buffer [R03722-D50]) and incubated with anti-Myc antibody (Abcam, ab18185) to detect CRYs. In all Western blot analyses, the blots were incubated overnight with a primary antibody. The murine IgGк-bind-

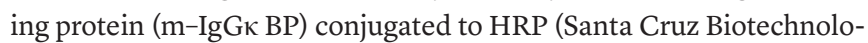
gy, sc-516102) was used as the secondary antibody. To capture the chemiluminescent signals, WesternBright ECL HRP substrate (Advansta, K-12045-D20) and Bio-Rad ChemiDoc Imaging System were used.

Docking with HADDOCK. The CLOCK-PASB domain (residues 261-384 from 4f3l.pdb) was docked into the secondary pocket of mCRY1 (PDB ID 5T5X) as described previously (36) via HADDOCK 2.2 server $(37,38)$. Active residues (directly involved in the interaction) for CRY were: G106, R109, E383, and E382; and for CLOCK PAS-B were: G332, H360, Q361, W362, and E367. Passive residues were defined automatically around the active residues. Docking was performed with default parameters.

Study approval. For the Turkish and Italian cohorts, we obtained written informed consent from all of the study participants at the time of blood sampling. The institutional ethics committee of Bilkent University and Siena University approved the study. A code and family ID number deidentified each individual. The consent procedure allows recontact for the collection of individual-level phenotypic data, which are different from the primary reason for referral.

Statistics. The subjects were classified into the following behavioral categories: (a) affected hyperactive/impulsive; (b) affected inattentive; (c) affected combined; (d) ADHD spectrum (probably not affected); (e) ADHD spectrum (probably affected); (f) not affected; and $(\mathrm{g})$ unknown/uninterpretable. They were also classified according to the severity of ADHD as severe, moderate, mild, or in partial remission (Supplemental Table 1). For the statistical analysis of the association between ADHD and CRY1 allele status, the first, second, third, and fourth categories were combined as "affected," and the fifth and sixth categories were combined as "unaffected." Subjects deemed "unknown/uninterpretable" were excluded from the analysis. The statistical analyses were performed using SPSS, version 23 (GraphPad Prism, version 6.0e, GraphPad Software) and in-house Perl scripts. Normality of the data was assessed with a Shapiro-Wilk test. For categorical variables, a Fisher's exact test, OR, and 95\% CI were calculated. Since the data were not normally distributed, a Mann-Whitney $U$ test was used for comparison of the groups. A 2-tailed, unpaired $t$ test was used for statistical evaluation of the CRY1 rescue and degradation assays. For further information, refer to Supplemental Table 24.

\section{Author contributions}

TO conceived of the project. IHK designed the functional characterization studies of the mutants and WT human CRY1. OEO, MEK, SG, KB, YW, AO, CA, ANB, MAT, AG, CF, AR, JLC, YI, CEA, MCS, IHK, and TO collected and analyzed the data. MCS, CA, MAT, and AG, board-certified psychiatrists, conducted psychiatric evaluations of the study participants. For the families living outside of Ankara, CA, MCS, OEO, and TO traveled to the families' hometowns in Konya (DSPD-1), Urfa (DSPD-4), and Kayseri (DSPD-6) to perform clinical evaluations. CA, MCS, MAT, and AG reviewed the ASRS questionnaires, and TO, OEO, MAT, and AG reviewed the MCTQs completed by each participant. TO, OEO, MAT, and 
AG conducted independent sleep behavior assessments. TO and IHK wrote the manuscript with input from all authors.

\section{Acknowledgments}

We are indebted to İclal Büyükdevrim Özçelik, Nezahat Doğan, and Eyyüp-Emel Göllü for their dedication at every step of the project and for their insightful communications with the families. We gratefully acknowledge Jeffrey M. Friedman, Aziz Sancar, Michael W. Young, Alina Patke, and Turgay Dalkara for their invaluable comments on the manuscript. We thank Andrew C. Liu and Hiroki Ueda for the gift of the $\mathrm{Cry}^{-/-}$and $\mathrm{Cry} 2^{-/-}$MEFs and the Cry 1 rescue vector. The Turkish Academy of Sciences-TÜBA supported this work. WES of the replication cohort was performed at the Yale Center for Mendelian Genomics, funded by the National Human Genome Research Institute and the National Heart, Lung, and Blood Institute (NHLBI), NIH (UM1HG006504). The GSP Coordinating Center (U24 HG008956) contributed to the cross-program scientific initiatives and provided logistical and general study coordination. This work was funded in part by the National Center for Advancing Translational Sciences (NCAST); the NIH Clinical and Translational Science Award (CTSA) program (UL1TR001866); the NIH
(R01AI088364, R01AI127564, R37AI095983, P01AI61093); the French National Research Agency (ANR) under the "Investments for the future" program (ANR-10-IAHU-01); the Integrative Biology of Emerging Infectious Diseases Laboratoire d'Excellence (ANR-10LABX-62-IBEID); an IEIHSEER grant (ANR-14-CE14-0008-01); a SEAe-Host Factors grant (ANR-18-CE15-0020 02); a PNEUMOID project grant (ANR 14-CE15-0009-01); and by grants from the INCA/Cancéropole Ile-de-France (2013-1-PL BIO-11-INSERM 5-1); the Rockefeller University, INSERM; the HHMI, University of Paris; the St. Giles Foundation; and the Charles Bronfman Institute for Personalized Medicine at the Icahn School of Medicine at Mount Sinai. The content is solely the responsibility of the authors and does not necessarily represent the official views of the NIH. CF and AR of this publication are members of the European Reference Network for rare malformation syndromes and rare intellectual and neurodevelopmental disorders, ERN-ITHACA.

Address correspondence to: Tayfun Özçelik, Bilkent University, Department of Molecular Biology and Genetics, Bilkent, Ankara 06800, Turkey. Phone: 90.312.266.4380; Email: tozcelik@ bilkent.edu.tr.
1. Patke A, et al. Mutation of the human circadian clock gene CRY1 in familial delayed sleep phase disorder. Cell. 2017;169(2):203-215.e13.

2. Toh KL, et al. An hPer2 phosphorylation site mutation in familial advanced sleep phase syndrome. Science. 2001;291(5506):1040-1043

3. Xu Y, et al. Functional consequences of a CKIdelta mutation causing familial advanced sleep phase syndrome. Nature. 2005;434(7033):640-644.

4. Hirano A, et al. A cryptochrome 2 mutation yields advanced sleep phase in humans. Elife. 2016;5:e16695.

5. Rahman SA, Kayumov L, Tchmoutina EA, Shap iro CM. Clinical efficacy of dim light melatonin onset testing in diagnosing delayed sleep phase syndrome. Sleep Med. 2009;10(5):549-555

6. Thapar A, Cooper M. Attention deficit hyperactivity disorder. Lancet. 2016;387(10024):1240-1250.

7. Wulff K, Gatti S, Wettstein JG, Foster RG. Sleep and circadian rhythm disruption in psychiatric and neurodegenerative disease. Nat Rev Neurosci. 2010;11(8):589-599.

8. Sehgal A, Mignot E. Genetics of sleep and sleep disorders. Cell. 2011;146(2):194-207.

9. Pagani L, et al. Genetic contributions to circadian activity rhythm and sleep pattern phenotypes in pedigrees segregating for severe bipolar disorder. Proc Natl Acad Sci USA. 2016;113(6):E754-E761.

10. Lane JM, et al. Genome-wide association analyses of sleep disturbance traits identify new loci and highlight shared genetics with neuropsychiatric and metabolic traits. Nat Genet. 2017;49(2):274-281.

11. Brainstorm Consortium, et al. Analysis of shared heritability in common disorders of the brain. Science. 2018;360(6395):eaap8757.

12. Demontis D, et al. Discovery of the first genomewide significant risk loci for attention deficit/hyperactivity disorder. Nat Genet. 2019;51(1):63-75.

13. Sullivan PF, Geschwind DH. Defining the genetic, genomic, cellular, and diagnostic architectures of psychiatric disorders. Cell. 2019;177(1):162-183.

14. McClellan J, King MC. Genetic heterogeneity in human disease. Cell. 2010;141(2):210-217.

15. Schulze TG, McMahon FJ. Defining the phenotype in human genetic studies: forward genetics and reverse phenotyping. Hum Hered. 2004;58(3-4):131-138.

16. Stessman HA, Bernier R, Eichler EE. A genotype-first approach to defining the subtypes of a complex disease. Cell. 2014;156(5):872-877.

17. Dal GM, et al. Early postzygotic mutations contribute to de novo variation in a healthy monozygotic twin pair. J Med Genet. 2014;51(7):455-459.

18. Özçelik T, Onat OE. Genomic landscape of the Greater Middle East. Nat Genet. 2016;48(9):978-979.

19. Ye R, Selby CP, Chiou YY, Ozkan-Dagliyan I, Gaddameedhi S, Sancar A. Dual modes of CLOCK:BMAL1 inhibition mediated by cryptochrome and period proteins in the mammalian circadian clock. Genes Dev. 2014;28(18):1989-1998.

20. American Psychiatric Association. Diagnostic and Statistical Manual of Mental Disorders. 5th ed. Washington, DC, USA: American Psychiatric Association; 2012

21. Roenneberg T, Wirz-Justice A, Merrow M. Life between clocks: daily temporal patterns of human chronotypes. J Biol Rhythms. 2003;18(1):80-90.

22. Adler LA, et al. Validity of pilot adult ADHD SelfReport Scale (ASRS) to rate adult ADHD symptoms. Ann Clin Psychiatry. 2006;18(3):145-148.

23. Bron TI, Bijlenga D, Verduijn J, Penninx BW, Beekman AT, Kooij JJ. Prevalence of ADHD symptoms across clinical stages of major depressive disorder. J Affect Disord. 2016;197:29-35.

24. Global Tobacco Surveillance System Data (GTSSData). Centers for Disease Control and Prevention Web Site. https://nccd.cdc.gov/GTSSDataSurveyResources/Ancillary/DataReports. aspx?CAID=1. Updated August 6, 2019. Accessed
September 17, 2019

25. Rybak YE, McNeely HE, Mackenzie BE, Jain UR, Levitan RD. An open trial of light therapy in adult attention-deficit/hyperactivity disorder. JClin Psychiatry. 2006;67(10):1527-1535.

26. Fargason RE, et al. Correcting delayed circadian phase with bright light therapy predicts improvement in ADHD symptoms: A pilot study. J Psychiatr Res. 2017;91:105-110.

27. Arns M, van der Heijden KB, Arnold LE, Kenemans JL. Geographic variation in the prevalence of attention-deficit/hyperactivity disorder: the sunny perspective. Biol Psychiatry. 2013;74(8):585-590.

28. Insel TR. The NIMH Research Domain Criteria (RDoC) Project: precision medicine for psychiatry. Am J Psychiatry. 2014;171(4):395-397.

29. Hennekam RC, Biesecker LG. Next-generation sequencing demands next-generation phenotyping. Hum Mutat. 2012;33(5):884-886.

30. Lee S, Abecasis GR, Boehnke M, Lin X. Rare-variant association analysis: study designs and statistical tests. Am J Hum Genet. 2014;95(1):5-23.

31. Karczewski KJ, et al. Variation across 141,456 human exomes and genomes reveals the spectrum of loss-of-function intolerance across human protein-coding genes. Posted on bioRxiv April 8, 2020. https://doi.org/10.1101/531210.

32. The 1000 Genomes Project Consortium. A map of human genome variation from population-scale sequencing. Nature. 2010;467(7319):1061-1073

33. Lazaridis I, et al. Genomic insights into the origin of farming in the ancient Near East. Nature. 2016;536(7617):419-424.

34. Green M, et al. Diagnosis of Attention-Deficit/Hyperactivity Disorder. Rockville Maryland, USA: Agency for Health Care Policy and Research; 1999.

35. Qiu M, Ramulu PY, Boland MV. Association between sleep parameters and glaucoma in the United States population: National Health and 
Nutrition Examination Survey. J Glaucoma. 2019;28(2):97-104.

36. Michael AK, et al. Formation of a repressive complex in the mammalian circadian clock is mediated by the secondary pocket of CRY1. Proc Natl Acad Sci USA. 2017;114(7):1560-1565.

37. Dominguez C, Boelens R, Bonvin AM. HADDOCK: a protein-protein docking approach based on biochemical or biophysical information. JAm Chem Soc. 2003;125(7):1731-1737.

38. van Zundert GCP, et al. The HADDOCK2.2 Web server: user-friendly integrative modeling of biomolecular complexes. J Mol Biol. 2016;428(4):720-725.

39. Ukai-Tadenuma M, Yamada RG, Xu H, Ripperger JA, Liu AC, Ueda HR. Delay in feedback repression by cryptochrome 1 is required for circadian clock function. Cell. 2011;144(2):268-281.

40. Siepka SM, et al. Circadian mutant overtime reveals F-box protein FBXL3 regulation of cryptochrome and period gene expression. Cell. 2007;129(5):1011-1023.

41. Gao P, et al. Phosphorylation of the cryptochrome $1 \mathrm{C}$-terminal tail regulates circadian period length. J Biol Chem. 2013;288(49):35277-35286.

42. Posner J, Polanczyk GV, Sonuga-Barke E. Attention-deficit hyperactivity disorder. Lancet. 2020;395(10222):450-462.

43. Sancar A. Mechanisms of DNA Repair by photolyase and excision nuclease (Nobel Lecture). Angew Chem Int Ed Engl. 2016;55(30):8502-8527.

44. Manolio TA, et al. Bedside back to bench: building bridges between basic and clinical genomic research. Cell. 2017;169(1):6-12.

45. Li H, Durbin R. Fast and accurate short read alignment with Burrows-Wheeler transform. Bioinformatics. 2009;25(14):1754-1760.

46. McKenna A, et al. The Genome Analysis Toolkit: a MapReduce framework for analyzing next-gen- eration DNA sequencing data. Genome Res. 2010;20(9):1297-1303.

47. Purcell S, et al. PLINK: a tool set for whole-genome association and population-based linkage analyses. Am JHum Genet. 2007;81(3):559-575.

48. Manichaikul A, Mychaleckyj JC, Rich SS, Daly K, Sale M, Chen WM. Robust relationship inference in genome-wide association studies. Bioinformatics. 2010;26(22):2867-2873.

49. Patterson N, Price AL, Reich D. Population structure and eigenanalysis. PLoS Genet. 2006;2(12):e190.

50. Cingolani $\mathrm{P}$, et al. A program for annotating and predicting the effects of single nucleotide polymorphisms, SnpEff: SNPs in the genome of Drosophila melanogaster strain w1118; iso-2; iso-3. Fly (Austin). 2012;6(2):80-92.

51. Wang K, Li M, Hakonarson H. ANNOVAR: functional annotation of genetic variants from high-throughput sequencing data. Nucleic Acids Res. 2010;38(16):e164.

52. Glusman G, Caballero J, Mauldin DE, Hood L, Roach JC. Kaviar: an accessible system for testing SNV novelty. Bioinformatics. 2011;27(22):3216-3217.

53. NHLBI GO Exome Sequencing Project (ESP). Exome Variant Server. http://evs.gs.washington.edu/EVS. Updated April 23, 2019. Accessed May 4, 2020.

54. Dong C, et al. Comparison and integration of deleteriousness prediction methods for nonsynonymous SNVs in whole exome sequencing studies. Hum Mol Genet. 2015;24(8):2125-2137.

55. Kircher M, Witten DM, Jain P, O'Roak BJ, Cooper GM, Shendure J. A general framework for estimating the relative pathogenicity of human genetic variants. Nat Genet. 2014;46(3):310-315.

56. Jian X, Liu X. In silico prediction of deleteriousness for nonsynonymous and splice-altering single nucleotide variants in the human genome.
Methods Mol Biol. 2017;1498:191-197.

57. Xiong HY, et al. RNA splicing. The human splicing code reveals new insights into the genetic determinants of disease. Science. 2015;347(6218):1254806

58. Ciarleglio CM, et al. Genetic differences in human circadian clock genes among worldwide populations. J Biol Rhythms. 2008;23(4):330-340.

59. Hawkins GA, Meyers DA, Bleecker ER, Pack AI. Identification of coding polymorphisms in human circadian rhythm genes PER1, PER2, PER3, CLOCK, ARNTL, CRY1, CRY2 and TIMELESS in a multi-ethnic screening panel. DNA Seq. 2008;19(1):44-49.

60. Delaneau O, Marchini J, Zagury JF. A linear complexity phasing method for thousands of genomes. Nat Methods. 2011;9(2):179-181.

61. Machiela MJ, Chanock SJ. LDlink: a web-based application for exploring population-specific haplotype structure and linking correlated alleles of possible functional variants. Bioinformatics. 2015;31(21):3555-3557.

62. Reeve JP, Rannala B. DMLE+: Bayesian linkage disequilibrium gene mapping. Bioinformatics. 2002;18(6):894-895.

63. Borroni B, et al. Founder effect and estimation of the age of the Progranulin Thr272fs mutation in 14 Italian pedigrees with frontotemporal lobar degeneration. Neurobiol Aging. 2011;32(3):555.e1-555.e8.

64. Ottoni C, Ricaut FX, Vanderheyden N, Brucato N, Waelkens M, Decorte R. Mitochondrial analysis of a Byzantine population reveals the differential impact of multiple historical events in South Anatolia. Eur J Hum Genet. 2011;19(5):571-576.

65. Maddison A. The World Economy: A Millennial Perspective. Paris, France: Development Centre of the Organisation for Economic Co-operation and Development; 2001. 